
\title{
Ruthenium-Catalyzed Heterocyclizations
}

Formation of Indoles, Dihydroisoquinolines and Dihydroquinolines by

\author{
Alejandro Varela-Fernández, Jesús A. Varela, Carlos Saá*
}

Departamento de Química Orgánica y Centro Singular de Investigación en Química Biológica y Materiales Molecular Universidad de Santiago de Compostela, 15782 Santiago de Compostela, Spain

Fax: (+34)-88-181-5704

E-mail: carlos.saa@usc.es

Received: The date will be inserted once the manuscript is accepted.

This article may be used for non-commercial purposes in accordance with Thieme Terms and Conditions for self-archiving.

Dedication - If you wish to insert a short dedication please overwrite this text, otherwise delete the paragraph.

Abstract: Indoles, dihydroisoquinolines and dihydroquinolines were efficiently prepared by ruthenium-catalyzed heterocyclizations of aromatic homo- and bis-homopropargyl amines/amides in the presence of an amine/ammonium baseacid pair. These regioselective 5- and 6-endo cyclizations most probably occur by nucleophilic trapping of key Ru vinylidene intermediates.

Key words: heterocyclizations; indoles; isoquinolines; quinolines; ruthenium; vinylidenes.<smiles>C#Cc1cc(C(C)=O)c[c]c1N</smiles><smiles>C#Cc1ccccc1CNC</smiles>

$3 g$<smiles></smiles>

5

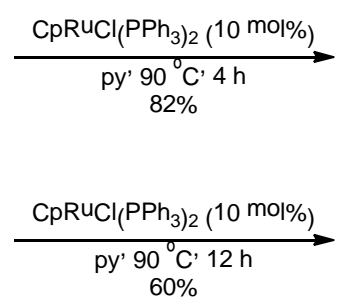
$60 \%$

Dihydrojsoquinolines<smiles>[I-]N1C=Cc2ccccc2C1</smiles>

$4 \mathrm{~g}$<smiles>CN1C=CCc2ccccc21</smiles>

Dihydroquinolines

Scheme 1 Ruthenium-Catalyzed Heterocyclization of Aromatic Homo- and Bis-Homopropargylic Amines/Amides: Formation of Indoles, Dihydroisoquinolines and Dihydroquinolines

\section{Introduction}

The development of new methods for the synthesis of heterocyclic compounds continues to be a major challenge in modern organic synthesis. ${ }^{1}$ During the last few years, many transition metal-catalyzed heterocyclizations have been developed to achieve these goals. ${ }^{2}$ Specifically, the nucleophilic trapping of metal vinylidene intermediates derived from terminal acetylenic compounds has been very succesful. ${ }^{3}$ Oxygenated heterocycles, e.g. dihydrofurans and dihydropyrans, were prepared in a pioneer work developed by McDonald and coworkers from heterocyclizations (cycloisomerizations) of homo- and bis-homopropargylic alcohols using catalytic Mo and $\mathrm{W}$ vinylidenes. ${ }^{4}$ More advances in similar hetero- and oxidative cyclizations were later developed by Trost and Rhee using ruthenium ${ }^{5}$ and rhodium catalysts. ${ }^{6}$ Seven-membered oxepines were recently synthesized by MacDonald and coworkers from heterocyclizations of preorganized alkynols using catalytic W vinylidenes. $^{7} \quad$ The corresponding benzofused derivatives, e.g. benzofurans and isochromenes, were synthesized by our own group on having used the regioselective 5-endo and 6-endo ruthenium-catalyzed heterocyclization of aromatic homo- and bishomopropargylic alcohols. ${ }^{8}$ More recently, even the more challenging seven-membered benzoxepines could also be synthesized by regioselective 7-endo heterocyclization of aromatic alkynols using in this case osmium catalysts. ${ }^{9}$ In the case of nitrogenated heterocycles, much effort has been devoted mainly to the synthesis of indoles. Since the pioneer work of McDonald using molybdenum catalysts, ${ }^{10}$ modern syntheses of indoles by heterocylization of substituted (2-ethynyl)anilines have been also described in Trost and Grotjahn groups using rhodium ${ }^{11}$ and ruthenium catalysts. ${ }^{12}$ Herein, we wish to report typical practical procedures, not only for the ruthenium-catalyzed synthesis of indoles, but to the six-membered 1,2dihydroisoquinolines and 1,4-dihydroquinolines by heterocyclization of aromatic homo- and bishomopropargyl amines/amides using commercial ruthenium catalysts. ${ }^{13}$ 


\section{Scope and limitations}

The ruthenium-catalyzed 5-endo heterocyclization of 2-(ethynyl)anilines $\mathbf{1}$ to indoles $\mathbf{2}$ was accomplished by heating at $90{ }^{\circ} \mathrm{C}$ a solution of the corresponding ethynylaniline 1 and $10 \mathrm{~mol} \%$ of commercial $\mathrm{CpRu}\left(\mathrm{PPh}_{3}\right)_{2} \mathrm{Cl}$ in pyridine (Table 1). The cyclization tolerates an unprotected amino group 1a and also electron-withdrawing substituents at the amino function, $\mathbf{1 b}$ and $\mathbf{1 c}$, with similar yields being obtained (Table 1, entries 1-3). The tolerance of the cyclization was excellent with the different functional groups tested (nitro 1d, nitrile 1e, ester $\mathbf{1 f , g}$ ) and gave goodto-excellent yields of the corresponding indoles $\mathbf{2 d}-\mathbf{g}$ (entries 4-7). Interestingly, cyclization seems more affected with the acid/base properties of the aniline rather than the electrophilicity of the vinylidene intermediates (entries 6 and 7). Internal alkyne such as 2-(hexyn-1-yl)aniline $\mathbf{1 h}$ was not suitable for this reaction (entry 8), which seems to indicate that the cyclization does not involve simple alkyne activation with the catalyst.

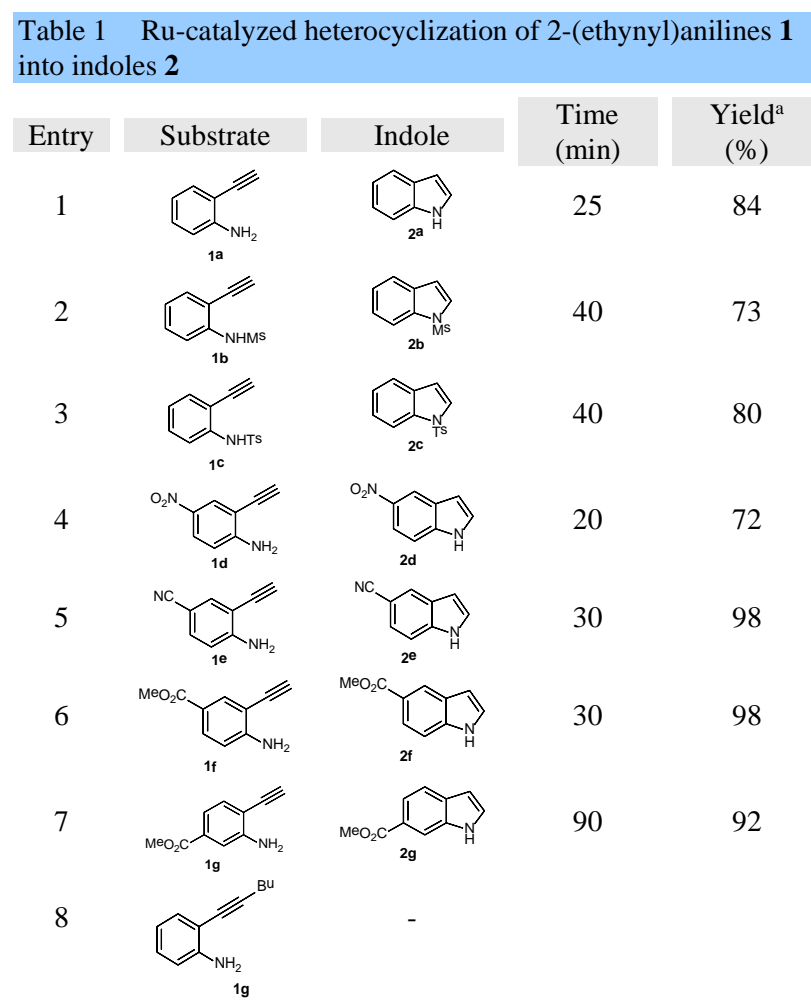

a Isolated yields. In all cases, conversion is higher than 97\% (GC monitoring).

The same procedure could be applied to the construction of 1,2-dihydroisoquinolines $\mathbf{4}$ from amide derivatives of 2-alkynylbenzylamines 3 (Table $3)$. Even though the parent benzylamine $3 \mathbf{a}$ and its secondary derivatives $N$-(methyl) and $N$ (phenyl)benzylamines failed to undergo heterocyclization, thus showing the important effect of the nature of the coordinating heteroatom, benzamide 3b underwent smooth regioselective 6-endo heterocyclization to give isoquinolone $\mathbf{4 b}$ in fairly good isolated yield (entry 2). Furthermore, cyclization of secondary benzamide 3c also gave isoquinolone 4c in quite good yield, although a longer reaction time was required (entry 3). To further highlight the unique synthetic potential of this cycloisomerization, the pharmaceutically active chromophore benzothiazine 1,1-dioxide 4d (benzosultam) was satisfactorily obtained from acyclic sulphonamide 3d (entry 4). By contrast, substituted benzamide $3 \mathbf{e}$ was not suitable for this reaction (entry 5), which again seems to indicate that the cyclization is not due to simple alkyne activation with the catalyst. Acetamide $\mathbf{3 f}$ and tosylamide $\mathbf{3 g}$ were smoothly cyclized to 1,2dihydroisoquinolines $\mathbf{4 f}$ and $\mathbf{4 g}$, thus showing the versatility of the amide nucleophile in the substrates (entries 6 and 7).

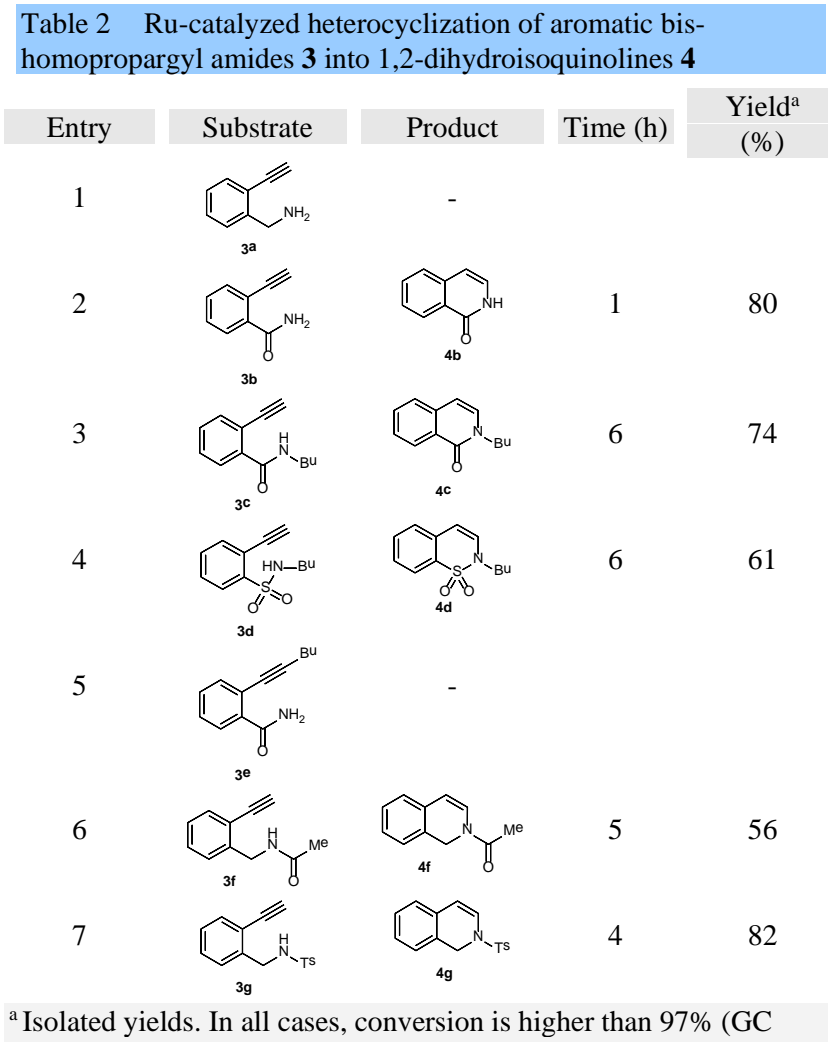

${ }^{a}$ Isolated yields. In all cases, conversion is higher than 97\% (GC monitoring.

Interestingly, the isomeric $2 H$-1,4-dihydroquinoline $\mathbf{6}$ could also be prepared by 6-endo heterocyclization of unsubstituted 2-(2-propynyl)tosylanilide $\mathbf{5}$ in quite reasonable 60\% yield (Scheme 2 ).

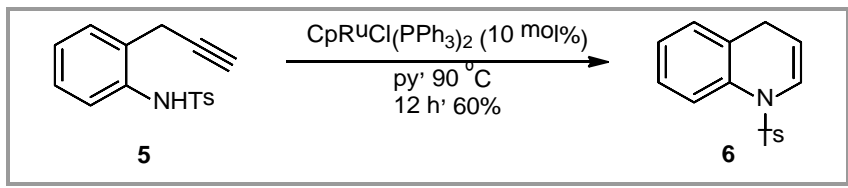

Scheme 2 Ru-catalyzed heterocyclization of aromatic bishomopropargylic amide 5 into 1,4-dihydroquinoline 6.

In summary, efficient Ru-catalyzed synthesis of indoles, 1,2-dihydroisoquinolines and 1,4dihydroquinoline by heterocyclization of aromatic 
homo- and bis-homopropargyl amines/amides have been developed. Most probably, these regioselective processes (5- and 6-endo cyclizations) occur by nucleophilic trapping of key $\mathrm{Ru}$ vinylidene intermediates. The presence of an amine/ammonium base-acid pair accelerates the cyclization and facilitates the catalytic turnover. The new procedures to synthesize 1,2-dihydroisoquinolines and 1,4dihydroquinoline by $\mathrm{C}-\mathrm{N}$ bond formation significantly increase the scope of metal vinylidene intermediates in catalytic processes.

\section{Procedures}

All reactions were carried out under an argon atmosphere in flame-dried glassware with magnetic stirring, unless otherwise noted. All starting materials were purchased from commercial suppliers and used without further purification, unless otherwise stated. Solvents were dried by distillation over an appropriate desiccant agent: tetrahydrofuran (THF) and diethyl ether $\left(\mathrm{Et}_{2} \mathrm{O}\right)$ were continuously refluxed and freshly distilled from sodium benzophenone ketyl under Ar; triethylamine $\left(\mathrm{Et}_{3} \mathrm{~N}\right)$, pyridine (py) and dichloromethane $\left(\mathrm{CH}_{2} \mathrm{Cl}_{2}\right)$ were continuously refluxed and freshly distilled from calcium hydride. Thin-layer chromatography (TLC) was carried out on silicacoated aluminium plates (silica gel $60 \quad \mathrm{~F}_{254}$ Merck) using UV light as visualizing agent (256 and $360 \mathrm{~nm}$ ) and cerium molybdate (Hanessian's stain, solution of $12 \mathrm{~g}$ of ammonium molybdate, $0.5 \mathrm{~g}$ of ceric ammonium molybdate and $15 \mathrm{~mL}$ of conc. sulfuric acid in $235 \mathrm{~mL}$ of water), $\mathrm{KMnO}_{4}$ (solution of $1.5 \mathrm{~g}$ of potassium permanganate, $10 \mathrm{~g}$ of potassium bicarbonate and $1.25 \mathrm{~mL}$ of $10 \%$ sodium hydroxide in $200 \mathrm{~mL}$ of water) or $p$-anisaldehyde (solution of 3.7 $\mathrm{mL}$ of $p$-anisaldehyde, $1.5 \mathrm{~mL}$ of glacial acetic acid, 5 $\mathrm{mL}$ of conc. sulfuric acid in $135 \mathrm{~mL}$ of absolute ethanol) with heat as developing agents. Flash chromatography was performed on silica gel 60 (Merck, 230-400 mesh) with the indicated eluent. ${ }^{1} \mathrm{H}$ and ${ }^{13} \mathrm{C}$ NMR were recorded on Bruker DPX-250 $\mathrm{MHz}, \mathrm{AMX}-300 \mathrm{MHz}, \mathrm{WM}-500 \mathrm{MHz}$ and Varian Inova-400 $\mathrm{MHz}$ instruments and chemical shifts are reported relative to tetramethylsilane as an internal reference. Coupling constants $J$ are given in Hertz $(\mathrm{Hz})$. Multiplicities are reported as follows: $\mathrm{s}=$ singlet, $\mathrm{d}=$ doublet, $\mathrm{t}=$ triplet, $\mathrm{q}=$ quartet, $\mathrm{m}=$ multiplet or as a combination of them. Multiplicities of ${ }^{13} \mathrm{C}$ NMR signals were determined by DEPT experiments. Gas chromatography (GC-MS) was recorded on an Agilent HP-6890N with a mass detector HP-5973N using a chemical ionization (CI) technique. High-resolution mass spectra (HRMS) was recorded on a Micromass Autospec spectrometer using CI (Chemical Ionization) or EI (Electron Ionization) techniques. Yields refer to isolated compounds estimated to be $>95 \%$ pure as determined by ${ }^{1} \mathrm{H}$ NMR and capillary GC analysis.

\section{Starting materials}

\section{General procedure for Sonogashira cross couplings}

Procedure 1:

The corresponding alkyne (1.50 equiv) was added to a suspension of aryl iodide (1 equiv), $\mathrm{PdCl}_{2}\left(\mathrm{PPh}_{3}\right)_{2}$ (0.01 equiv) and $\mathrm{CuI}$ (0.03 equiv) in a 3:1 mixture of $\mathrm{THF} / \mathrm{Et}_{3} \mathrm{~N}(0.1 \mathrm{M})$. The reaction mixture was stirred at room temperature until disappearance of starting material (TLC and GC-MS monitoring). The mixture was filtered through silica gel and the filtrate was evaporated to dryness. The residue was dissolved in EtOAc and washed twice with brine. The combined organic layers were dried over anhydrous $\mathrm{Na}_{2} \mathrm{SO}_{4}$, filtered and evaporated under vacuum. The residue was purified by flash column chromatography through silica gel using a mixture of EtOAc/Hex as eluent to afford the corresponding cross coupling product.

\section{Procedure 2:}

The corresponding alkyne (1.50 equiv) was added to a suspension of aryl bromide (1 equiv), $\mathrm{Pd}(\mathrm{OAc})_{2}(0.05$ equiv), CuI (0.05 equiv) and $\mathrm{PPh}_{3}$ (0.1 equiv) in $\mathrm{Et}_{3} \mathrm{~N}$ $(0.1 \mathrm{M})$. The reaction mixture was heated at $90{ }^{\circ} \mathrm{C}$ for $24 \mathrm{~h}$ until disappearance of starting material (TLC and GC-MS monitoring). The mixture was filtered through silica gel and the filtrate was evaporated to dryness. The residue was dissolved in EtOAc and washed twice with brine. The combined organic layers were dried over anhydrous $\mathrm{Na}_{2} \mathrm{SO}_{4}$, filtered and evaporated under vacuum. The residue was purified by flash column chromatography through silica gel using a mixture of EtOAc/Hex as eluent to afford the corresponding cross coupling product.

\section{General procedure for desilylation of (trimethylsilyl)ethynyl derivatives.}

A solution of TBAF in THF (1M, 1.5 equiv) was added dropwise to a solution of (trimethylsilyl)ethynyl derivative (1 equiv) in THF. The reaction mixture was stirred at room temperature until disappearance of starting material (TLC and GC-MS monitoring). The reaction was quenched by adding brine and extracted three times with EtOAc. The combined organic layers were dried over anhydrous $\mathrm{Na}_{2} \mathrm{SO}_{4}$, filtered and evaporated under vacuum. The residue was purified by flash column chromatography through silica gel using a mixture of $\mathrm{EtOAc/Hex}$ as eluent to give the corresponding desilylated product.

\section{2-Ethynylaniline (1a)}

The general Sonogashira cross coupling procedure 1 was followed using iodoaniline $(0.60 \mathrm{~g}, 2.74 \mathrm{mmol})$, $\mathrm{PdCl}_{2}\left(\mathrm{PPh}_{3}\right)_{2}(0.019 \mathrm{~g}, 0.027 \mathrm{mmol})$, CuI (0.016 g, $0.082 \mathrm{mmol})$, trimethylsilylacetylene $(0.40 \mathrm{~g}, 0.58$ $\mathrm{mL}, 4.11 \mathrm{mmol})$, THF $(20 \mathrm{~mL})$ and $\mathrm{Et}_{3} \mathrm{~N}(7 \mathrm{~mL})$. Upon completion (4 h) and work-up, the residue was purified by flash column chromatography through silica gel using a 1:4 mixture of EtOAc/Hex as eluent 
to give 2-[(trimethylsilyl)ethynyl]aniline (0.52 g, 99\%) as a brown oil.

${ }^{1} \mathrm{H}$ NMR (250 MHz, CDCl3): $\delta=7.26$ (d, $J=7.7 \mathrm{~Hz}$, $1 \mathrm{H}), 7.05$ (t, $J=7.7,1 \mathrm{H}), 6.61$ (t, $J=7.7 \mathrm{~Hz}, 2 \mathrm{H})$, 4.18 (s, 2H), 0.24 (s, 9H)).

${ }^{13} \mathrm{C}$ NMR, DEPT (62 MHz, CDCl3): $\delta=148.1$ (C), $132.0(\mathrm{CH}), 129.8(\mathrm{CH}), 117.6(\mathrm{CH}), 114.1(\mathrm{CH})$, 107.5 (C), 101.8 (C), 99.5 (C), 0.0 (3 x CH3).

MS (CI): m/z (\%) = $190\left(\mathrm{M}^{+}+1,100\right), 174$ (52), 73 (20).

HRMS-CI: $\mathrm{m} / \mathrm{z}$ calcd for $\mathrm{C}_{11} \mathrm{H}_{16} \mathrm{NSi} \quad\left[\mathrm{M}^{+}+1\right]$ : 190.1052; found: 190.1052 .

The general desilylation procedure was followed using 2-[(trimethylsilyl)ethynyl]aniline (0.49 g, 2.59 mmol), a solution of TBAF in THF (1M, $3.89 \mathrm{~mL})$ and THF (25 mL). Upon completion (10 min) and work-up, the residue was purified by flash column chromatography through silica gel using a 1:4 mixture of EtOAc/Hex as eluent to give 1a $(0.29 \mathrm{~g}, 97 \%)$ as a brown oil.

${ }^{1} \mathrm{H}$ NMR (250 MHz, $\left.\mathrm{CDCl}_{3}\right): \delta=7.31$ (d, $J=7.7 \mathrm{~Hz}$, $1 \mathrm{H}), 7.13$ (t, $J=7.7 \mathrm{~Hz}, 1 \mathrm{H}), 6.71-6.62$ (m, 2H), 4.23 (s, 2H), 3.37 (s, 1H).

${ }^{13} \mathrm{C}$ NMR, DEPT (62 MHz, $\left.\mathrm{CDCl}_{3}\right): \delta=148.5$ (C), $132.5(\mathrm{CH}), 130.1(\mathrm{CH}), 117.7(\mathrm{CH}), 114.2(\mathrm{CH})$, $106.5(\mathrm{C}), 82.4(\mathrm{CH}), 80.6(\mathrm{C})$.

MS (CI): m/z (\%) = $118\left(\mathrm{M}^{+}+1,100\right), 91$ (2).

HRMS-CI: $\mathrm{m} / \mathrm{z}$ calcd for $\mathrm{C}_{8} \mathrm{H}_{8} \mathrm{~N}\left[\mathrm{M}^{+}+1\right]$ : 118.0657 ; found: 118.0653 .

\section{$N-[2-$ \\ [(trimethylsilyl)ethynyl]phenyl]methanesulfonamid e (1b)}

A solution of methanesulfonyl chloride $(0.57 \mathrm{~g}, 0.39$ $\mathrm{mL}, 5.02 \mathrm{mmol})$ in THF (2.5 mL) was added dropwise to a solution of iodoaniline ( $1 \mathrm{~g}, 4.56 \mathrm{mmol})$, pyridine (0.76 g, $0.77 \mathrm{~mL}, 9.58 \mathrm{mmol})$ in THF (10 mL) under Ar. The solution was stirred at room temperature for $12 \mathrm{~h}$ until disappearance of starting material (TLC, GC-MS monitoring). The resulting mixture was evaporated under vacuum and the residue was purified by flash column chromatography through silica gel using a 2:8 mixture of EtOAc/Hex as eluent to afford $N$-(2-iodophenyl)methanesulfonamide (1.22 g, 90\%) as a yellowish solid.

${ }^{1} \mathrm{H}$ NMR (250 MHz, $\left.\mathrm{CDCl}_{3}\right): \delta=7.82(\mathrm{~d}, J=7.7 \mathrm{~Hz}$, 1H), 7.63 (d, $J=7.9 \mathrm{~Hz}, 1 \mathrm{H}), 7.37$ (t, $J=7.4 \mathrm{~Hz}, 1 \mathrm{H})$ $6.93(\mathrm{t}, J=7.44 \mathrm{~Hz}, 1 \mathrm{H}), 6.74$ (s, br, 1H), 3.02 (s, $3 \mathrm{H})$.

${ }^{13} \mathrm{C}$ NMR, DEPT (62 MHz, $\left.\mathrm{CDCl}_{3}\right): \delta=139.3(\mathrm{CH})$, 137.5 (C), 129.7 (CH), $127.2(\mathrm{CH}), 122.5(\mathrm{CH}), 92.2$ (C), $40.1\left(\mathrm{CH}_{3}\right)$.

MS (EI, $70 \mathrm{eV}): \mathrm{m} / \mathrm{z}(\%)=297\left(\mathrm{M}^{+}, 17\right), 218$ (49), 170 (5), 108 (21), 91 (100).

The general Sonogashira cross coupling procedure 1 was followed using $\mathrm{N}$-(2- iodophenyl)methanesulfonamide (1.20 g, $4.04 \mathrm{mmol})$, $\mathrm{PdCl}_{2}\left(\mathrm{PPh}_{3}\right)_{2}$ (0.028 g, $\left.0.040 \mathrm{mmol}\right)$, CuI (0.023 g, $0.12 \mathrm{mmol})$, trimethylsilylacetylene $(0.59 \mathrm{~g}, 0.85 \mathrm{~mL}$, $6.06 \mathrm{mmol})$, THF (30 mL) and $\mathrm{Et}_{3} \mathrm{~N}(10 \mathrm{~mL})$. Upon completion (12 h) and work-up, the residue was purified by flash column chromatography through silica gel using a 1:5 mixture of EtOAc/Hex as eluent to give N-(2((trimethylsilyl)ethynyl)phenyl)methanesulfonamide (1.02 g, $95 \%)$ as a colorless solid.

${ }^{1} \mathrm{H}$ NMR (300 MHz, $\mathrm{CDCl}_{3}$ ): $\delta=7.58$ (d, $J=7.7 \mathrm{~Hz}$, $1 \mathrm{H}), 7.47$ (dd, J = 7.7, $1.4 \mathrm{~Hz}, 1 \mathrm{H}), 7.36$ (td, $\mathrm{J}=7.7$, $1.4 \mathrm{~Hz}, 1 \mathrm{H}), 7.12$ (td, J = 7.7, $1.4 \mathrm{~Hz}, 1 \mathrm{H}), 7.00$ (s, br, $1 \mathrm{H}), 3.00$ (s, 3H), 0.29 (s, 9H).

MS (EI, $70 \mathrm{eV}): \mathrm{m} / \mathrm{z}(\%)=267\left(\mathrm{M}^{+}, 69\right), 252$ (100).

HRMS-EI: $\mathrm{m} / \mathrm{z}$ calcd for $\mathrm{C}_{12} \mathrm{H}_{17} \mathrm{NO}_{2} \mathrm{SSi}\left[\mathrm{M}^{+}\right]$: 267.0750; found: 267.0719.

The general desilylation procedure was followed using N-(2((trimethylsilyl)ethynyl)phenyl)methanesulfonamide (1.00 g, $3.74 \mathrm{mmol})$, a solution of TBAF in THF (1M, $5.61 \mathrm{~mL}$ ) and THF (30 mL). Upon completion (10 min) and work-up, the residue was purified by flash column chromatography through silica gel using a 3:7 mixture of EtOAc/Hex as eluent to give $\mathbf{1 b}$ (0.62 g, $85 \%$ ) as a yellow solid.

${ }^{1} \mathrm{H}$ NMR (300 MHz, $\left.\mathrm{CDCl}_{3}\right) \delta$ (ppm): 7.61 (d, $J=$ $8.26 \mathrm{~Hz}, 1 \mathrm{H}), 7.51$ (d, $J=7.66 \mathrm{~Hz}, 1 \mathrm{H}), 7.39$ (t, $J=$ $7.84 \mathrm{~Hz}, 1 \mathrm{H}), 7.14$ (t, $J=7.58 \mathrm{~Hz}, 1 \mathrm{H}), 7.03$ (s, 1H), 3.50 (s, 1H), 3.03 (s, 3H).

${ }^{13} \mathrm{C}$ NMR, DEPT (75 MHz, $\left.\mathrm{CDCl}_{3}\right), \delta$ (ppm): 138.6 (C), $132.8(\mathrm{CH}), 130.5(\mathrm{CH}), 124.7(\mathrm{CH}), 119.5(\mathrm{CH})$, $112.9(\mathrm{C}), 84.9(\mathrm{CH}), 78.6(\mathrm{C}), 39.7\left(\mathrm{CH}_{3}\right)$.

HRMS-EI: m/z calcd for $\mathrm{C}_{9} \mathrm{H}_{9} \mathrm{NO}_{2} \mathrm{~S}\left[\mathrm{M}^{+}\right]$: 195.0354 ; found: 195.0354 .

\section{4-methyl- $N$-[2- \\ [(trimethylsilyl)ethynyl]phenyl]benzenesulfonamid e (1c)}

A solution of 4-methylbenzene-1-sulfonyl chloride (3.97 g, $20.54 \mathrm{mmol})$ in THF (10 mL) was added dropwise over $2 \mathrm{~h}$ to a solution of iodoaniline (4.50 g, $20.54 \mathrm{mmol})$, pyridine (1.70 g, $1.74 \mathrm{~mL}, 21.54 \mathrm{mmol})$ in THF (30 mL) under Ar. The solution was stirred at room temperature for $12 \mathrm{~h}$ until disappearance of starting material (TLC, GC-MS monitoring). The resulting mixture was evaporated under vacuum and the residue was purified by flash column chromatography through silica gel using a 1:9 mixture of EtOAc/Hex as eluent to afford $N$-(2-iodophenyl)-4methylbenzenesulfonamide $(7.50 \mathrm{~g}, 98 \%)$ as a white solid.

${ }^{1} \mathrm{H}$ NMR (250 MHz, $\left.\mathrm{CDCl}_{3}\right): \delta=7.60$ (m, 4H), 7.28 (td, J = 8.2, 1.3 Hz, 1H), 7.19 (d, J = 8.2 Hz, 2H), 6.88 (s, 1H), 6.80 (td, J = 7.7, 1.3 Hz, 1H), 2.34 (s, 3H).

${ }^{13} \mathrm{C}$ NMR, DEPT (62 MHz, $\left.\mathrm{CDCl}_{3}\right): \delta=144.22(\mathrm{C})$, 139.09 (CH), 137.40 (C), 135.79 (C), 129.63 (2xCH), 
$129.45(\mathrm{CH}), 127.39$ (2xCH), $126.90(\mathrm{CH}), 122.50$ (CH), $92.47(\mathrm{C}), 21.58\left(\mathrm{CH}_{3}\right)$.

MS (CI): m/z (\%) = $374\left(\mathrm{M}^{+}+1,100\right), 247$ (92), 246 (25), 219 (18).

The general Sonogashira cross coupling procedure 1 was followed using $N$-(2-iodophenyl)-4methylbenzenesulfonamide (3.00 g, $8.05 \mathrm{mmol})$, $\mathrm{PdCl}_{2}\left(\mathrm{PPh}_{3}\right)_{2}$ (0.056 g, $\left.0.080 \mathrm{mmol}\right)$, CuI (0.045 g, $0.024 \mathrm{mmol})$, trimethylsilylacetylene $(1.18 \mathrm{~g}, 1.70$ $\mathrm{mL}, 12.06 \mathrm{mmol})$, THF $(60 \mathrm{~mL})$ and $\mathrm{Et}_{3} \mathrm{~N}(20 \mathrm{~mL})$. Upon completion (12 h) and work-up, the residue was purified by flash column chromatography through silica gel using a 2:8 mixture of EtOAc/Hex as eluent to give 4-methyl- $N$-(2((trimethylsilyl)ethynyl)phenyl)benzenesulfonamide ( $2.51 \mathrm{~g}, 91 \%$ ) as a yellow solid.

${ }^{1} \mathrm{H}$ NMR $\left(250 \mathrm{MHz}, \mathrm{CDCl}_{3}\right): \delta=7.35(\mathrm{t}, \mathrm{J}=8.4 \mathrm{~Hz}$, 3H), 6.98 (m, 3H), 6.90 (d, J = 8.4 Hz, 2H), 6.70 (t, J $=7.6 \mathrm{~Hz}, 1 \mathrm{H}), 2.06(\mathrm{~s}, 3 \mathrm{H}), 0.008(\mathrm{~s}, 9 \mathrm{H})$.

${ }^{13} \mathrm{C}$ NMR, DEPT (62 MHz, $\left.\mathrm{CDCl}_{3}\right): \delta=144.01$ (C), 138.07 (C), 135.98 (C), $131.96(\mathrm{CH}), 129.81(\mathrm{CH})$, $129.58(2 \mathrm{xCH}), 127.20(2 \mathrm{xCH}), 124.33(\mathrm{CH}), 119.72$ (CH), 114.19 (C), $102.23(\mathrm{C}), 99.51(\mathrm{C}), 21.52\left(\mathrm{CH}_{3}\right)$, $-0.13\left(3 \mathrm{xCH}_{3}\right)$.

MS (CI): m/z (\%) = $344\left(\mathrm{M}^{+}+1,100\right), 328$ (19).

The general desilylation procedure was followed using 4-methyl- $N$-(2((trimethylsilyl)ethynyl)phenyl)benzenesulfonamide (1.79 g, $5.22 \mathrm{mmol})$, a solution of TBAF in THF (1M, $7.82 \mathrm{~mL})$ and THF $(50 \mathrm{~mL})$. Upon completion (10 min) and work-up, the residue was purified by flash column chromatography through silica gel using a 2:8 mixture of EtOAc/Hex as eluent to give 1c (1.30 g, $92 \%)$ as a yellow oil.

${ }^{1} \mathrm{H}$ NMR $\left(250 \mathrm{MHz}, \mathrm{CDCl}_{3}\right): \delta=7.70(\mathrm{~d}, \mathrm{~J}=8.2 \mathrm{~Hz}$, $2 \mathrm{H}), 7.60(\mathrm{~d}, \mathrm{~J}=8.2 \mathrm{~Hz}, 1 \mathrm{H}), 7.33(\mathrm{t}, \mathrm{J}=7.7 \mathrm{~Hz}, 2 \mathrm{H})$, 7.24 (d, J = 8.2 Hz, 2H), $7.02(\mathrm{t}, \mathrm{J}=7.7 \mathrm{~Hz}, 1 \mathrm{H}), 3.30$ (s, 1H), 2.37 (s, 3H).

${ }^{13} \mathrm{C}$ NMR, DEPT (62 MHz, $\mathrm{CDCl}_{3}$ ): $\delta=144.22$ (C), 138.52 (C), 135.98 (C), 132.59 (CH), $130.24(\mathrm{CH})$, $129.74(2 \mathrm{xCH}), 127.42(2 \mathrm{xCH}), 124.26(\mathrm{CH}), 119.37$ $(\mathrm{CH}), 112.76(\mathrm{C}), 84.55(\mathrm{CH}), 78.66(\mathrm{C}), 21.65\left(\mathrm{CH}_{3}\right)$.

MS (CI): m/z (\%) = $272\left(\mathrm{M}^{+}+1,100\right), 118$ (21).

\section{4-Nitro-2-[(trimethylsilyl)ethynyl]aniline (1d)}

The general Sonogashira cross coupling procedure 1 was followed using 2-iodo-4-nitroaniline $(0.60 \mathrm{~g}, 2.27$ $\mathrm{mmol}), \mathrm{PdCl}_{2}\left(\mathrm{PPh}_{3}\right)_{2}$ (0.016 g, $\left.0.023 \mathrm{mmol}\right), \mathrm{CuI}$ $(0.013 \mathrm{~g}, 0.069 \mathrm{mmol})$, trimethylsilylacetylene $(0.33$ g, $0.48 \mathrm{~mL}, 3.40 \mathrm{mmol})$, THF $(16 \mathrm{~mL})$ and $\mathrm{Et}_{3} \mathrm{~N}(6$ $\mathrm{mL})$. Upon completion (12 h) and work-up, the residue was purified by flash column chromatography through silica gel using a 1:4 mixture of EtOAc/Hex as eluent to give 4-nitro-2[(trimethylsilyl)ethynyl+aniline (0.49 g, $93 \%)$ as a yellow solid.
${ }^{1} \mathrm{H}$ NMR (250 MHz, $\left.\mathrm{CDCl}_{3}\right): \delta=8.22(\mathrm{~d}, J=2.6 \mathrm{~Hz}$, $1 \mathrm{H}$ ), 8.01 (dd, $J=9.0,2.6 \mathrm{~Hz}, 1 \mathrm{H}), 6.67$ (d, $J=9.0$ $\mathrm{Hz}, 1 \mathrm{H}), 4.96$ (s, 2H), 0.28 (s, 9H).

${ }^{13} \mathrm{C}$ NMR, DEPT (62 MHz, $\left.\mathrm{CDCl}_{3}\right): \delta=153.3$ (C), $138.1(\mathrm{C}), 128.9(\mathrm{CH}), 126.1(\mathrm{CH}), 112.7(\mathrm{CH}), 106.9$ (C), 102.1 (C), 98.9 (C), -0.1 (3 x CH$)_{3}$.

MS (CI): m/z (\%) = $235\left(\mathrm{M}^{+}+1,100\right), 219$ (69), 205 (83), 189 (52), 73 (35).

HRMS-CI: $\mathrm{m} / \mathrm{z}$ calcd for $\mathrm{C}_{11} \mathrm{H}_{15} \mathrm{~N}_{2} \mathrm{O}_{2} \mathrm{Si}\left[\mathrm{M}^{+}+1\right]$ : 235.0903; found: 235.0903.

The general desilylation procedure was followed using 4-nitro-2-[(trimethylsilyl)ethynyl]aniline (0.40 g, $1.71 \mathrm{mmol})$, a solution of TBAF in THF (1M, 2.56 $\mathrm{mL})$ and THF $(15 \mathrm{~mL})$. Upon completion (10 min) and work-up, the residue was purified by flash column chromatography through silica gel using a 1:4 mixture of EtOAc/Hex as eluent to give $\mathbf{1 d ~ ( 0 . 1 8 ~ g , ~ 6 3 \% ) ~ a s ~ a ~}$ yellow solid.

${ }^{1} \mathrm{H}$ NMR (300 MHz, $\left.\mathrm{CDCl}_{3}\right): \delta=8.25$ (s, $\left.1 \mathrm{H}\right), 8.04$ (d, $J=9.0 \mathrm{~Hz}, 1 \mathrm{H}), 6.68$ (d, $J=9.0 \mathrm{~Hz}, 1 \mathrm{H}), 5.00$ (s, 2H), $3.47(\mathrm{~s}, 1 \mathrm{H})$.

${ }^{13} \mathrm{C}$ NMR, DEPT (75 MHz, $\left.\mathrm{CDCl}_{3}\right): \delta=153.5$ (C), 137.3 (C), $129.3(\mathrm{CH}), 126.4(\mathrm{CH}), 112.9(\mathrm{CH}), 105.7$ (C), $84.2(\mathrm{CH}), 78.1(\mathrm{C})$.

MS (EI, $70 \mathrm{eV}): \mathrm{m} / \mathrm{z}(\%)=162\left(\mathrm{M}^{+}, 100\right), 132(40)$, 116 (24), 89 (97), 63 (45), 58 (35).

HRMS-EI: m/z calcd for $\mathrm{C}_{8} \mathrm{H}_{6} \mathrm{~N}_{2} \mathrm{O}\left[\mathrm{M}^{+}\right]$: 162.0429; found: 162.0429 .

\section{4-Amino-3-ethynylbenzonitrile (1e)}

The general Sonogashira cross coupling procedure 1 was followed using 4-amino-3-iodobenzonitrile (1.50 g, $6.15 \mathrm{mmol}), \mathrm{PdCl}_{2}\left(\mathrm{PPh}_{3}\right)_{2}(0.043 \mathrm{~g}, 0.061 \mathrm{mmol})$, CuI $(0.035 \mathrm{~g}, 0.18 \mathrm{mmol})$, trimethylsilylacetylene (0.91 g, $1.30 \mathrm{~mL}, 9.22 \mathrm{mmol}$ ), THF (45 mL) and $\mathrm{Et}_{3} \mathrm{~N}$ (15 mL). Upon completion (12 h) and work-up, the residue was purified by flash column chromatography through silica gel using a 1:4 mixture of EtOAc/Hex as eluent to give 4-amino-3[(trimethylsilyl)ethynyl]benzonitrile (1.18 g, $90 \%)$ as a yellow solid.

${ }^{1} \mathrm{H}$ NMR $\left(250 \mathrm{MHz}, \mathrm{CDCl}_{3}\right): \delta=7.56(\mathrm{~d}, J=1.8 \mathrm{~Hz}$, $1 \mathrm{H}), 7.33$ (dd, $J=8.5,1.8 \mathrm{~Hz}, 1 \mathrm{H}), 6.68$ (d, $J=8.5$ Hz, 1H), 4.79 (s, 2H), 0.27 (s, 9H).

${ }^{13} \mathrm{C}$ NMR, DEPT (62 MHz, $\left.\mathrm{CDCl}_{3}\right): \delta=151.4$ (C), 136.4 (CH), $133.3(\mathrm{CH}), 119.3(\mathrm{C}), 113.8(\mathrm{CH}), 107.9$ (C), 102.0 (C), 99.7 (C), 98.9 (C), - 0.1 (3 x CH 3 ).

MS (CI): m/z (\%) = $215\left(\mathrm{M}^{+}+1,100\right), 199$ (70), 73 (17).

HRMS-CI: $\mathrm{m} / \mathrm{z}$ calcd for $\mathrm{C}_{12} \mathrm{H}_{15} \mathrm{~N}_{2} \mathrm{Si} \quad\left[\mathrm{M}^{+}+1\right]$ : 215.1005; found: 215.1005.

The general desilylation procedure was followed using 4-amino-3-[(trimethylsilyl)ethynyl]benzonitrile $(0.80 \mathrm{~g}, 3.74 \mathrm{mmol})$, a solution of TBAF in THF (1M, $3.60 \mathrm{~mL}$ ) and THF (35 mL). Upon completion (10 min) and work-up, the residue was purified by flash 
column chromatography through silica gel using a 3:7 mixture of EtOAc/Hex as eluent to give 1e $(0.45 \mathrm{~g}$, $84 \%)$ as a yellow solid.

${ }^{1} \mathrm{H}$ NMR (300 MHz, $\left.\mathrm{CDCl}_{3}\right): \delta=7.57$ (s, $\left.1 \mathrm{H}\right), 7.36$ (d, $J=8.5 \mathrm{~Hz}, 1 \mathrm{H}), 6.70$ (d, $J=8.5 \mathrm{~Hz}, 1 \mathrm{H}), 4.85$ (s, 2H), 3.46 (s, 1H).

${ }^{13} \mathrm{C}$ NMR, DEPT (75 MHz, $\left.\mathrm{CDCl}_{3}\right): \delta=151.8$ (C), $136.8(\mathrm{CH}), 133.5(\mathrm{CH}), 119.2(\mathrm{C}), 114.0(\mathrm{CH}), 106.6$ (C), 99.6 (C), $84.2(\mathrm{CH}), 78.1(\mathrm{C})$.

MS (EI, $70 \mathrm{eV}): \mathrm{m} / \mathrm{z}(\%)=142\left(\mathrm{M}^{+}, 100\right), 115$ (66), 62 (18).

HRMS-EI: $\mathrm{m} / \mathrm{z}$ calcd for $\mathrm{C}_{9} \mathrm{H}_{6} \mathrm{~N}_{2}\left[\mathrm{M}^{+}\right]$: 142.0531; found: 142.0531 .

\section{Methyl 4-amino-3-ethynylbenzoate (1f)}

The general Sonogashira cross coupling procedure 1 was followed using methyl 4-amino-3-iodobenzoate (1.00 g, $3.61 \mathrm{mmol}), \mathrm{PdCl}_{2}\left(\mathrm{PPh}_{3}\right)_{2}$ (0.025 g, 0.036 $\mathrm{mmol})$ CuI (0.021 g, $0.11 \quad \mathrm{mmol})$, trimethylsilylacetylene $(0.53 \mathrm{~g}, 0.77 \mathrm{~mL}, 5.42 \mathrm{mmol})$, THF (27 mL) and Et $3 \mathrm{~N}$ (9 mL). Upon completion (12 h) and work-up, the residue was purified by flash column chromatography through silica gel using a 3:7 mixture of EtOAc/Hex as eluent to give methyl 4amino-3-[(trimethylsilyl)ethynyl]benzoate (0.78 g, 87 $\%)$ as a yellow solid.

${ }^{1} \mathrm{H}$ NMR (250 MHz, $\left.\mathrm{CDCl}_{3}\right): \delta=8.00$ (s, 1H), 7.78 (d, $J=8.6 \mathrm{~Hz}, 1 \mathrm{H}), 6.65$ (d, $J=8.6 \mathrm{~Hz}, 1 \mathrm{H}), 4.68$ (s, 2H), 3.84 (s, 3H), 0.26 (s, 9H).

${ }^{13} \mathrm{C}$ NMR, DEPT (62 MHz, $\left.\mathrm{CDCl}_{3}\right): \delta=166.5(\mathrm{C}=\mathrm{O})$, 151.8 (C), $134.5(\mathrm{CH}), 131.5(\mathrm{CH}), 119.1(\mathrm{C}), 113.1$ (CH), 106.9 (C), $100.5(\mathrm{C}), 100.4(\mathrm{C}), 51.7\left(\mathrm{CH}_{3}\right)$, $0.1\left(3 \times \mathrm{CH}_{3}\right)$.

MS (CI): m/z (\%) = $248\left(\mathrm{M}^{+}+1,100\right), 232$ (28), 185 (26), 144 (17), 57 (64).

HRMS-CI: $\mathrm{m} / \mathrm{z}$ calcd for $\mathrm{C}_{13} \mathrm{H}_{18} \mathrm{NO}_{2} \mathrm{Si}\left[\mathrm{M}^{+}+1\right]$ : 248.1107; found: 248.1107.

The general desilylation procedure was followed using methyl 4-amino-3[(trimethylsilyl)ethynyl]benzoate $(0.49 \mathrm{~g}, 2.00 \mathrm{mmol})$, a solution of TBAF in THF (1M, $3.00 \mathrm{~mL})$ and THF (20 mL). Upon completion (10 min) and work-up, the residue was purified by flash column chromatography through silica gel using a 1:4 mixture of EtOAc/Hex as eluent to give 1f (0.34 g, 97\%) as a yellow solid.

${ }^{1} \mathrm{H}$ NMR (300 MHz, $\left.\mathrm{CDCl}_{3}\right): \delta=8.03$ (s, 1H), 7.80 (d, $J=8.6,1 \mathrm{H}), 6.67$ (d, $J=8.6 \mathrm{~Hz}, 1 \mathrm{H}), 4.76$ (s, 2H), 3.84 (s, 3H), 3.41 (s, 1H).

${ }^{13} \mathrm{C}$ NMR, DEPT (75 MHz, $\left.\mathrm{CDCl}_{3}\right): \delta=166.4(\mathrm{C}=\mathrm{O})$, 152.2 (C), $134.8(\mathrm{CH}), 131.7(\mathrm{CH}), 119.0(\mathrm{C}), 113.2$ (CH), $105.6(\mathrm{C}), 82.9(\mathrm{CH}), 79.4(\mathrm{C}), 51.6\left(\mathrm{CH}_{3}\right)$.

MS (CI): m/z (\%) = $176\left(\mathrm{M}^{+}+1,100\right), 144$ (12).

HRMS-EI: $\mathrm{m} / \mathrm{z}$ calcd for $\mathrm{C}_{10} \mathrm{H}_{9} \mathrm{NO}_{2}\left[\mathrm{M}^{+}\right]$: 175.0633 ; found: 175.0633 .

\section{Methyl 3-amino-4-ethynylbenzoate (1g)}

The general Sonogashira cross coupling procedure 1 was followed using methyl 3-amino-4-iodobenzoate (1.00 g, $3.61 \mathrm{mmol}), \mathrm{PdCl}_{2}\left(\mathrm{PPh}_{3}\right)_{2}(0.025 \mathrm{~g}, 0.036$ $\mathrm{mmol})$, $\mathrm{CuI} \quad(0.021 \mathrm{~g}, \quad 0.11 \mathrm{mmol})$, trimethylsilylacetylene $(0.53 \mathrm{~g}, 0.77 \mathrm{~mL}, 5.42 \mathrm{mmol})$, THF (27 mL) and Et ${ }_{3} \mathrm{~N}$ (9 mL). Upon completion (12 h) and work-up, the residue was purified by flash column chromatography through silica gel using a 3:7 mixture of EtOAc/Hex as eluent to give methyl 3amino-4-((trimethylsilyl)ethynyl)benzoate (0.86 g, 90 $\%)$ as a colorless solid.

${ }^{1} \mathrm{H}$ NMR (500 MHz, $\left.\mathrm{CDCl}_{3}\right): \delta=7.30-7.36$ (m, 3H), 4.34 (s, 2H), 3.88 (s, 3H), 0.27 (s, 9H.

${ }^{13} \mathrm{C}$ NMR (125 MHz, $\left.\mathrm{CDCl}_{3}\right): \delta=166.8(\mathrm{C}=\mathrm{O})$, 148.1, 132.2, 131.0, 118.6, 114.9, 112.0, 102.7, 100.9, 52.1, 0.00 .

The general desilylation procedure was followed using methyl 3-amino-4((trimethylsilyl)ethynyl)benzoate (0.49 g, $2.00 \mathrm{mmol})$, a solution of TBAF in THF (1M, $3.00 \mathrm{~mL})$ and THF (20 mL). Upon completion (10 min) and work-up, the residue was purified by flash column chromatography through silica gel using a 1:4 mixture of EtOAc/Hex as eluent to give $1 \mathrm{~g}(0.33 \mathrm{~g}, 95 \%)$ as a yellow solid.

${ }^{1} \mathrm{H}$ NMR (300 MHz, $\left.\mathrm{CDCl}_{3}\right): \delta=7.40-7.29$ (m, 3H), 4.41 (s, br, 2H), 3.88 (s, 3H), 3.52 (s, 1H).

${ }^{13} \mathrm{C}$ NMR (75 MHz, $\left.\mathrm{CDCl}_{3}\right): \delta=166.8(\mathrm{C}=\mathrm{O}), 148.4$, 132.5, 131.3, 118.5, 115.1, 110.7, 84.9, 79.9, 52.2.

MS (EI, $70 \mathrm{eV}): \mathrm{m} / \mathrm{z}(\%)=175\left(\mathrm{M}^{+}, 100\right), 144$ (46), 116 (22), 89 (14).

\section{2-(Hex-1-yn-1-yl)aniline (1h)}

The general Sonogashira cross coupling procedure 1 was followed using 2-iodoaniline (0.50 g, $2.28 \mathrm{mmol})$, $\mathrm{PdCl}_{2}\left(\mathrm{PPh}_{3}\right)_{2}$ (0.048 g, $\left.0.068 \mathrm{mmol}\right)$, CuI (0.026 g, $0.14 \mathrm{mmol})$, hex-1-yne (0.28 g, $0.39 \mathrm{~mL}, 3.42 \mathrm{mmol})$, THF (16 mL) and $\mathrm{Et}_{3} \mathrm{~N}$ (6 mL). Upon completion (12 h) and work-up, the residue was purified by flash column chromatography through silica gel using a 1:4 mixture of EtOAc/Hex as eluent to give 1h $(0.34 \mathrm{~g}, 87$ $\%)$ as a yellowish oil.

${ }^{1} \mathrm{H}$ NMR (250 MHz, $\mathrm{CDCl}_{3}$ ): $\delta=7.23$ (dd, $J=7.8,1.3$ $\mathrm{Hz}, 1 \mathrm{H}), 7.09-7.00$ (m, 1H), 6.69-6.58 (m, 2H), 4.13 (s, 2H), 2.44 (t, J = 6.9 Hz, 2H), 1.64-1.40 (m, 4H), $0.93(\mathrm{t}, J=7.1 \mathrm{~Hz}, 3 \mathrm{H})$.

${ }^{13} \mathrm{C}$ NMR, DEPT (62 MHz, $\left.\mathrm{CDCl}_{3}\right): \delta=147.5(\mathrm{C})$, $131.8(\mathrm{CH}), 128.6(\mathrm{CH}), 117.6(\mathrm{CH}), 114.0(\mathrm{CH})$, 108.7 (C), $95.5(\mathrm{C}), 76.9(\mathrm{C}), 30.8\left(\mathrm{CH}_{2}\right), 21.9\left(\mathrm{CH}_{2}\right)$, $19.1\left(\mathrm{CH}_{2}\right), 13.5\left(\mathrm{CH}_{3}\right)$.

MS (CI): m/z (\%) = $174\left(\mathrm{M}^{+}+1,100\right), 132$ (10), 57 (17).

HRMS-CI: $\mathrm{m} / \mathrm{z}$ calcd for $\mathrm{C}_{12} \mathrm{H}_{16} \mathrm{~N}\left[\mathrm{M}^{+}+1\right]$ : 174.1283; found: 174.1283 .

\section{(2-Ethynylphenyl)methanamine (3a)}

The general Sonogashira cross coupling procedure 1 was followed using 2-iodobenzonitrile (1.50 g, 6.55 mmol), $\mathrm{PdCl}_{2}\left(\mathrm{PPh}_{3}\right)_{2}$ (0.046 g, $\left.0.066 \mathrm{mmol}\right)$, CuI 
(0.038 g, $0.20 \mathrm{mmol})$, trimethylsilylacetylene (0.96 g, $1.39 \mathrm{~mL}, 9.82 \mathrm{mmol})$, THF $(48 \mathrm{~mL})$ and $\mathrm{Et}_{3} \mathrm{~N}(16$ $\mathrm{mL})$. Upon completion (12 h) and work-up, the residue was purified by flash column chromatography through silica gel using a 0.5:9.5 mixture of EtOAc/Hex as eluent to give 2[(trimethylsilyl)ethynyl]benzonitrile (1.29 g, $99 \%)$ as a brown oil.

${ }^{1} \mathrm{H}$ NMR $\left(250 \mathrm{MHz}, \mathrm{CDCl}_{3}\right): \delta=7.35-7.18(\mathrm{~m}, 3 \mathrm{H})$, 7.15-7.05 (m, 1H), 0.00 (s, 9H).

${ }^{13} \mathrm{C}$ NMR, DEPT (62 MHz, $\left.\mathrm{CDCl}_{3}\right): \delta=132.0(\mathrm{CH})$, $131.9(\mathrm{CH}), 131.8(\mathrm{CH}), 128.2(\mathrm{CH}), 126.2(\mathrm{C}), 116.6$ (C), $115.2(\mathrm{C}), 101.4(\mathrm{C}), 100.2(\mathrm{C}),-0.8\left(3 \mathrm{x} \mathrm{CH}_{3}\right)$.

MS (CI): m/z (\%) = $200\left(\mathrm{M}^{+}+1,100\right), 184$ (56), 128 (11).

The general desilylation procedure was followed using 2-[(trimethylsilyl)ethynyl]benzonitrile (1.00 g, $5.05 \mathrm{mmol})$, a solution of TBAF in THF (1M, 7.54 $\mathrm{mL}$ ) and THF $(50 \mathrm{~mL})$. Upon completion $(10 \mathrm{~min})$ and work-up, the residue was purified by flash column chromatography through silica gel using a 1:4 mixture of $\mathrm{EtOAc/Hex}$ as eluent to give 2-ethynylbenzonitrile (0.49 g, 76\%) as a white solid.

A solution of 2-ethynylbenzonitrile (0.20 g, 1.57 $\mathrm{mmol})$ in $\mathrm{Et}_{2} \mathrm{O}(0.70 \mathrm{~mL})$ was added dropwise into a suspension of $\mathrm{LiAlH}_{4}(0.12 \mathrm{~g}, 3.15 \mathrm{mmol})$ in $\mathrm{Et}_{2} \mathrm{O}$ $(2.30 \mathrm{~mL})$ at $-10^{\circ} \mathrm{C}$. The resultant slurry was stirred at $-10{ }^{\circ} \mathrm{C}$ for $1 \mathrm{~h}$ and, then, at room temperature for another $1 \mathrm{~h}$. The reaction was quenched by the careful dropwise addition of $\mathrm{H}_{2} \mathrm{O}(0.2 \mathrm{~mL})$ followed by a saturated solution of $\mathrm{NaOH}(0.2 \mathrm{~mL})$. The resultant biphasic mixture was stirred vigourously for $2 \mathrm{~h}$ at room temperature. The layers were then allowed to separate and the aqueous layer was extracted with additional $\mathrm{Et}_{2} \mathrm{O}(2 \mathrm{~mL})$. The combined organic layers were washed with brine $(15 \mathrm{~mL})$, dried (anhydrous $\mathrm{Na}_{2} \mathrm{SO}_{4}$ ), filtered over Celite and concentrated under vacuum to afford 3a (0.16 g, 76\%) as a brown oil.

${ }^{1} \mathrm{H}$ NMR $\left(250 \mathrm{MHz}, \mathrm{CDCl}_{3}\right): \delta=7.48(\mathrm{~d}, J=7.5 \mathrm{~Hz}$, $1 \mathrm{H})$, 7.34-7.28 (m, 2H), 7.23-7.16 (m, $1 \mathrm{H}), 3.96(\mathrm{~s}$, 2H), 3.32 (s, 1H), 1.61 (s, 2H).

${ }^{13} \mathrm{C}$ NMR, DEPT (62 MHz, $\left.\mathrm{CDCl}_{3}\right): \delta=145.8(\mathrm{C})$, $132.9(\mathrm{CH}), 129.1(\mathrm{CH}), 127.2(\mathrm{CH}), 126.6(\mathrm{CH})$, $120.5(\mathrm{C}), 82.7(\mathrm{C}), 81.5(\mathrm{CH}), 45.2\left(\mathrm{CH}_{2}\right)$.

MS (CI): m/z (\%) = $132\left(\mathrm{M}^{+}+1,100\right), 115$ (92).

\section{2-Ethynylbenzamide (3b)}

The general Sonogashira cross coupling procedure 2 was followed using 2-bromobenzamide (2.00 g, 10 $\mathrm{mmol}), \mathrm{Pd}(\mathrm{OAc})_{2}(0.11 \mathrm{~g}, 0.50 \mathrm{mmol})$, CuI (0.095 g, $0.50 \mathrm{mmol}), \quad \mathrm{PPh}_{3}(0.26 \mathrm{~g}, 1.00 \mathrm{mmol})$, trimethylsilylacetylene (1.47 g, $2.12 \mathrm{~mL}, 15 \mathrm{mmol})$ and $\mathrm{Et}_{3} \mathrm{~N}(80 \mathrm{~mL})$. Upon completion $(24 \mathrm{~h})$ and workup, the residue was purified by flash column chromatography through silica gel using a 2:3 mixture of EtOAc/Hex as eluent to give 2[(trimethylsilyl)ethynyl]benzamide (0.69 g, 32\%) as a yellow solid.
The general desilylation procedure was followed using 2-[(trimethylsilyl)ethynyl]benzamide $(0.60 \mathrm{~g}$, $2.76 \mathrm{mmol})$, a solution of TBAF in THF (1M, 4.15 $\mathrm{mL}$ ) and THF (25 mL). Upon completion (10 min) and work-up, the residue was purified by flash column chromatography through silica gel using a 1:1 mixture of EtOAc/Hex as eluent to give $3 \mathbf{b}(0.17 \mathrm{~g}, 42 \%)$ as a yellow solid.

${ }^{1} \mathrm{H}$ NMR (400 MHz, $\left.\mathrm{CDCl}_{3}\right): \delta=8.09-8.06(\mathrm{~m}, 1 \mathrm{H})$, 7.62-7.58 (m, 1H), 7.48-7.44 (m, 2H), $6.44(\mathrm{~s}, 2 \mathrm{H})$, $3.53(\mathrm{~s}, 1 \mathrm{H})$.

${ }^{13} \mathrm{C}$ NMR, DEPT $\left(100 \mathrm{MHz}, \mathrm{CDCl}_{3}\right): \delta=168.0$ $(\mathrm{C}=\mathrm{O}), 135.4(\mathrm{C}), 134.2(\mathrm{CH}), 130.9(\mathrm{CH}), 130.1$ (CH), $129.4(\mathrm{CH}), 118.9(\mathrm{C}), 83.9(\mathrm{CH}), 82.3(\mathrm{C})$.

MS (EI, $70 \mathrm{eV}): \mathrm{m} / \mathrm{z}(\%)=145\left(\mathrm{M}^{+}, 91\right), 129(40)$, 117 (35), 101 (80), 75 (56).

HRMS-EI: m/z calcd for $\mathrm{C}_{9} \mathrm{H}_{7} \mathrm{NO}\left[\mathrm{M}^{+}\right]$: 145.0528; found: 145.0528 .

\section{$\mathrm{N}$-butyl-2-ethynylbenzamide (3c)}

In a flamed flame-dried round-bottomed flask under Ar 2-iodobenzoic acid (1.49 g, $6.00 \mathrm{mmol})$ was introduced and then thionyl chloride $(7.14 \mathrm{~g}, 4.35 \mathrm{~mL}$, $60 \mathrm{mmol}$ ) was added dropwise. The mixture was stirred at room temperature for $12 \mathrm{~h}$ (NMR monitoring) until no gas emission was observed. Excess $\mathrm{SOCl}_{2}$ was removed in vacuo. The crude 2iodobenzoyl chloride (1.35 g, 84\%) as a white solid was then used without further purification.

${ }^{1} \mathrm{H}$ NMR (250 MHz, $\mathrm{CDCl}_{3}$ ): $\delta=8.12-8.00(\mathrm{~m}, 2 \mathrm{H})$, $7.50(\mathrm{~m}, J=7.6 \mathrm{~Hz}, 1 \mathrm{H}), 7.26(\mathrm{t}, J=7.6 \mathrm{~Hz}, 1 \mathrm{H})$.

${ }^{13} \mathrm{C}$ NMR, DEPT $\left(62 \mathrm{MHz}, \mathrm{CDCl}_{3}\right): \delta=167.0(\mathrm{C}=\mathrm{O})$, $141.9(\mathrm{CH}), 137.7(\mathrm{C}), 134.4(\mathrm{CH}), 133.4(\mathrm{CH}), 128.2$ (CH), $93.8(\mathrm{C})$.

A solution of 2-iodobenzoyl chloride (1.30 g, 4.89 $\mathrm{mmol})$ in $\mathrm{CH}_{2} \mathrm{Cl}_{2}(25 \mathrm{~mL})$ was added dropwise to a mixture of butan-1-amine $(0.32 \mathrm{~g}, 0.44 \mathrm{~mL}, 4.42$ $\mathrm{mmol}), \mathrm{Et}_{3} \mathrm{~N}(0.54 \mathrm{~g}, 0.74 \mathrm{~mL}, 5.33 \mathrm{mmol})$ in $\mathrm{CH}_{2} \mathrm{Cl}_{2}$ (25 mL) under Ar. The solution was stirred at room temperature for $12 \mathrm{~h}$ until disappearance of starting material (TLC, GC-MS monitoring). The resulting mixture was filtered by gravity, washed with $\mathrm{H}_{2} \mathrm{O}(2 \mathrm{x}$ $25 \mathrm{~mL}$ ), dried over anhydrous $\mathrm{Na}_{2} \mathrm{SO}_{4}$, filtered and evaporated under vacuum. The residue was purified by flash column chromatography through silica gel using a 1:9 mixture of EtOAc/Hex as eluent to afford N-butyl-2-iodobenzamide ( $0.75 \mathrm{~g}, 50 \%)$ as a yellow solid.

${ }^{1} \mathrm{H}$ NMR $\left(250 \mathrm{MHz}, \mathrm{CDCl}_{3}\right): \delta=7.83(\mathrm{~d}, J=7.9 \mathrm{~Hz}$, $1 \mathrm{H}), 7.35(\mathrm{~d}, J=4.3 \mathrm{~Hz}, 2 \mathrm{H}), 7.13-7.02(\mathrm{~m}, 1 \mathrm{H}), 5.93$ (s, $1 \mathrm{H}), 3.47-3.37$ (m, 2H), 1.68-1.54 (m, 2H), 1.51$1.35(\mathrm{~m}, 2 \mathrm{H}), 0.96(\mathrm{t}, J=7.3 \mathrm{~Hz}, 3 \mathrm{H})$.

${ }^{13} \mathrm{C}$ NMR, DEPT (62 MHz, $\left.\mathrm{CDCl}_{3}\right): \delta=169.3(\mathrm{C}=\mathrm{O})$, $142.4(\mathrm{C}), 139.7(\mathrm{CH}), 130.9(\mathrm{CH}), 128.1(\mathrm{CH}), 128.0$ (CH), $92.4(\mathrm{C}), 39.7\left(\mathrm{CH}_{2}\right), 31.4\left(\mathrm{CH}_{2}\right), 20.1\left(\mathrm{CH}_{2}\right)$, $13.7\left(\mathrm{CH}_{3}\right)$. 
The general Sonogashira cross coupling procedure 1 was followed using $N$-butyl-2-iodobenzamide $(0.70 \mathrm{~g}$, $2.31 \mathrm{mmol}), \mathrm{PdCl}_{2}\left(\mathrm{PPh}_{3}\right)_{2}(0.084 \mathrm{~g}, 0.12 \mathrm{mmol}), \mathrm{CuI}$ (0.023 g, $0.12 \mathrm{mmol})$, trimethylsilylacetylene (0.34 g, $0.49 \mathrm{~mL}, 3.46 \mathrm{mmol})$, THF (17 mL) and $\mathrm{Et}_{3} \mathrm{~N}(6 \mathrm{~mL})$. Upon completion (4 h) and work-up, the residue was purified by flash column chromatography through silica gel using a 3:7 mixture of EtOAc/Hex as eluent to give $N$-butyl-2-[(trimethylsilyl)ethynyl]benzamide (0.62 g, $98 \%$ ) as a yellowish oil.

${ }^{1} \mathrm{H}$ NMR (250 MHz, $\left.\mathrm{CDCl}_{3}\right): \delta=8.18-8.06(\mathrm{~m}, 1 \mathrm{H})$, 7.69 (s, 1H), 7.59-7.51 (m, 1H), 7.50-7.37 (m, 2H), 3.57-3.43 (m, 2H), 1.69-1.57 (m, 2H), 1.54-1.38 (m, 2H), 0.97 (t, $J=7.2 \mathrm{~Hz}, 3 \mathrm{H}), 0.29$ (s, 9H).

${ }^{13} \mathrm{C}$ NMR, DEPT (62 MHz, $\left.\mathrm{CDCl}_{3}\right): \delta=165.7(\mathrm{C}=\mathrm{O})$, 135.4 (C), 133.9 (CH), 130.2 (CH), $130.1(\mathrm{CH}), 129.0$ (CH), 119.1 (C), $103.6(\mathrm{C}), 101.4(\mathrm{C}), 39.8\left(\mathrm{CH}_{2}\right)$, $31.6\left(\mathrm{CH}_{2}\right), 20.3\left(\mathrm{CH}_{2}\right), 13.8\left(\mathrm{CH}_{3}\right),-0.3\left(3 \times \mathrm{CH}_{3}\right)$.

MS (CI): m/z (\%) = $274\left(\mathrm{M}^{+}+1,100\right), 194$ (23), 126 (41).

HRMS (ESI): $\mathrm{m} / \mathrm{z}$ calcd for $\mathrm{C}_{16} \mathrm{H}_{24} \mathrm{NOSi}\left[\mathrm{M}^{+}+1\right]$ : 274.1627; found: 274.1622 .

The general desilylation procedure was followed using $N$-butyl-2-[(trimethylsilyl)ethynyl]benzamide (0.60 g, $2.20 \mathrm{mmol})$, a solution of TBAF in THF (1M, $3.30 \mathrm{~mL})$ and THF (22 mL). Upon completion (10 min) and work-up, the residue was purified by flash column chromatography through silica gel using a 3:7 mixture of EtOAc/Hex as eluent to give 3c (0.27 g, $61 \%)$ as a yellow solid.

${ }^{1} \mathrm{H}$ NMR (250 MHz, $\left.\mathrm{CDCl}_{3}\right): \delta=7.92-7.87(\mathrm{~m} 1 \mathrm{H})$, 7.56-7.50 (m, 1H), 7.43-7.35 (m, 2H), 7.28 (s, 1H), 3.50 (s, 1H), 3.47-3.42 (m, 2H), 1.67-1.54 (m, 2H), 1.45-1.37 (m, 2H), 0.95 (t, $J=7.2 \mathrm{~Hz}, 3 \mathrm{H})$.

${ }^{13} \mathrm{C}$ NMR, DEPT (62 MHz, $\left.\mathrm{CDCl}_{3}\right): \delta=166.0(\mathrm{C}=\mathrm{O})$, $136.8(\mathrm{C}), 133.9(\mathrm{CH}), 130.0(\mathrm{CH}), 129.3(\mathrm{CH}), 129.0$ (CH), $118.2(\mathrm{C}), 83.1(\mathrm{CH}), 82.0(\mathrm{C}), 39.6\left(\mathrm{CH}_{2}\right), 31.1$ $\left(\mathrm{CH}_{2}\right), 20.0\left(\mathrm{CH}_{2}\right), 13.6\left(\mathrm{CH}_{3}\right)$.

MS (CI): m/z (\%) = $202\left(\mathrm{M}^{+}+1,100\right), 146$ (11), 126 (18), 110 (14), 85 (8).

HRMS (ESI): $\mathrm{m} / \mathrm{z}$ calcd for $\mathrm{C}_{13} \mathrm{H}_{16} \mathrm{NO}\left[\mathrm{M}^{+}+1\right]$ : 202.1232; found: 202.1226.

\section{N-butyl-2-ethynylbenzenesulfonamide (3d)}

Butan-1-amine (0.45 g, $0.61 \mathrm{~mL}, 6.18 \mathrm{mmol}$ ) was added dropwise to a solution of 2-bromobenzene-1sulfonyl chloride $(0.56 \mathrm{~g}, 2.21 \mathrm{mmol})$ in $\mathrm{CHCl}_{3}(20$ $\mathrm{mL}$ ) cooled at $0{ }^{\circ} \mathrm{C}$. The resulting solution was then stirred at room temperature for $2 \mathrm{~h}$ until disappearance of starting material (TLC, GC-MS monitoring). The reaction mixture was evaporated under vacuum and the residue was dissolved in $\mathrm{Et}_{2} \mathrm{O}(10 \mathrm{~mL})$, washed with brine ( 2 x $10 \mathrm{~mL}$ ), dried over anhydrous $\mathrm{Na}_{2} \mathrm{SO}_{4}$, filtered and evaporated under vacuum. The resulting residue was purified by flash column chromatography through silica gel using a 3:7 mixture of EtOAc/Hex as eluent to give 2-bromo- $N$-butylbenzenesulfonamide (0.52 g, 50\%) as a white solid.
${ }^{1} \mathrm{H}$ NMR (250 MHz, $\left.\mathrm{CDCl}_{3}\right): \delta=8.15(\mathrm{~d}, J=7.3 \mathrm{~Hz}$, $1 \mathrm{H}), 7.74$ (d, $J=7.4 \mathrm{~Hz}, 1 \mathrm{H}), 7.53-7.39$ (m, 2H), 5.21 (s, 1H), 2.91 (dd, $J=13.3,6.7 \mathrm{~Hz}, 2 \mathrm{H}), 1.52-1.22$ (m, $4 \mathrm{H}), 0.84$ (t, $J=7.2 \mathrm{~Hz}, 3 \mathrm{H})$.

${ }^{13} \mathrm{C}$ NMR, DEPT (62 MHz, $\left.\mathrm{CDCl}_{3}\right): \delta=138.6$ (C), $134.9(\mathrm{CH}), 133.6(\mathrm{CH}), 131.5(\mathrm{CH}), 127.7(\mathrm{CH})$, 119.5 (C), $43.0\left(\mathrm{CH}_{2}\right), 31.3\left(\mathrm{CH}_{2}\right), 20.0\left(\mathrm{CH}_{2}\right), 13.4$ $\left(\mathrm{CH}_{3}\right)$.

MS (CI): m/z (\%) = $292\left(\mathrm{M}^{+}+1,100\right), 238$ (13), 236 (13), 72 (7).

The general Sonogashira cross coupling procedure 2 was followed using 2-bromo- $N$ butylbenzenesulfonamide $(0.50 \mathrm{~g}, 1.72 \mathrm{mmol})$, $\mathrm{Pd}(\mathrm{OAc})_{2}$ (0.060 g, $\left.0.086 \mathrm{mmol}\right)$, CuI (0.016 g, 0.086 $\mathrm{mmol}), \quad \mathrm{PPh}_{3} \quad(0.045 \mathrm{~g}, \quad 0.017 \quad \mathrm{mmol})$, trimethylsilylacetylene $(0.253 \mathrm{~g}, 0.36 \mathrm{~mL}, 2.58 \mathrm{mmol})$ and $\mathrm{Et}_{3} \mathrm{~N}(17 \mathrm{~mL})$. Upon completion (12 h) and workup, the residue was purified by flash column chromatography through silica gel using a 1:4 mixture of EtOAc/Hex as eluent to give $N$-butyl-2[(trimethylsilyl)ethynyl]benzenesulfonamide (0.23 g, $43 \%)$ as a yellowish oil.

${ }^{1} \mathrm{H}$ NMR (250 MHz, $\mathrm{CDCl}_{3}$ ): $\delta=7.79$ (d, $J=7.3 \mathrm{~Hz}$, 1H), 7.41 (d, $J=7.5 \mathrm{~Hz}, 1 \mathrm{H}), 7.34-7.20$ (m, 2H), 5.09 (t, $J=6.0 \mathrm{~Hz}, 1 \mathrm{H}), 2.67-2.63(\mathrm{~m}, 2 \mathrm{H}), 1.30-1.00$ (m, 4H), 0.63 (t, $J=7.3 \mathrm{~Hz}, 3 \mathrm{H}), 0.08$ (s, 9H).

${ }^{13} \mathrm{C}$ NMR, DEPT (62 MHz, $\left.\mathrm{CDCl}_{3}\right): \delta=141.0$ (C), $134.3(\mathrm{CH}), 131.9(\mathrm{CH}), 128.8(\mathrm{CH}), 128.7(\mathrm{CH})$, 119.8 (C), 103.5 (C), 101.4 (C), $42.8\left(\mathrm{CH}_{2}\right), 31.2$ $\left(\mathrm{CH}_{2}\right), 19.6\left(\mathrm{CH}_{2}\right), 13.3\left(\mathrm{CH}_{3}\right),-0.6\left(3 \times \mathrm{CH}_{3}\right)$.

MS (CI): m/z (\%) = $310\left(\mathrm{M}^{+}+1,100\right), 294$ (20).

HRMS-CI: $\mathrm{m} / \mathrm{z}$ calcd for $\mathrm{C}_{15} \mathrm{H}_{24} \mathrm{NO}_{2} \mathrm{SSi}\left[\mathrm{M}^{+}+1\right]$ : 310.1297; found: 310.1298.

A solution of TBAF in THF (1M, $5 \mathrm{~mL})$ was added dropwise to a solution of $N$-butyl-2[(trimethylsilyl)ethynyl]benzenesulfonamide $(0.22 \mathrm{~g}$, $0.71 \mathrm{mmol})$ in THF $(5 \mathrm{~mL})$ cooled at $-78{ }^{\circ} \mathrm{C}$. The reaction mixture was stirred at $-78{ }^{\circ} \mathrm{C}$ for $2 \mathrm{~h}$ until disappearance of starting material (TLC and GC-MS monitoring). The reaction was quenched by adding a saturated solution of citric acid. The resulting mixture was extracted with $\mathrm{Et}_{2} \mathrm{O}(3 \times 5 \mathrm{~mL})$ and washed with $\mathrm{H}_{2} \mathrm{O}(2 \times 5 \mathrm{~mL})$ and a saturated solution of $\mathrm{NaHCO}_{3}$ $(2 \times 5 \mathrm{~mL})$. The combined organic layers were dried over anhydrous $\mathrm{Na}_{2} \mathrm{SO}_{4}$, filtered and evaporated under vacuum. The residue was purified by flash column chromatography through silica gel using a 1:4 mixture of EtOAc/Hex as eluent to afford 3d (0.15 g, 88\%) as a brown solid.

${ }^{1} \mathrm{H}$ NMR (250 MHz, $\left.\mathrm{CDCl}_{3}\right): \delta=8.08-8.02$ (m, 1H), 7.72-7.66 (m, 1H), 7.59-7.47 (m, 2H), $5.21(\mathrm{t}, J=5.6$ $\mathrm{Hz}, 1 \mathrm{H}), 3.66$ (s, 1H), 2.90 (dd, $J=13.3,6.6 \mathrm{~Hz}, 2 \mathrm{H}$ ), 1.52-1.24 (m, 4H), 0.85 (t, $J=7.2 \mathrm{~Hz}, 3 \mathrm{H})$.

${ }^{13} \mathrm{C}$ NMR, DEPT (62 MHz, $\left.\mathrm{CDCl}_{3}\right): \delta=141.4$ (C), $135.1(\mathrm{CH}), 132.0(\mathrm{CH}), 129.2(\mathrm{CH}), 129.1(\mathrm{CH})$, 119.2 (C), $85.7(\mathrm{CH}), 80.2(\mathrm{C}), 43.0\left(\mathrm{CH}_{2}\right), 31.4$ $\left(\mathrm{CH}_{2}\right), 19.6\left(\mathrm{CH}_{2}\right), 13.4\left(\mathrm{CH}_{3}\right)$. 
MS (CI): m/z (\%) = $238\left(\mathrm{M}^{+}+1,100\right), 182$ (15).

HRMS-CI: $\mathrm{m} / \mathrm{z}$ calcd for $\mathrm{C}_{12} \mathrm{H}_{16} \mathrm{NO}_{2} \mathrm{~S} \quad\left[\mathrm{M}^{+}+1\right]$ : 238.0902; found: 238.0896.

\section{2-(Hex-1-yn-1-yl)benzamide (3e)}

The general Sonogashira cross coupling procedure 2 was followed using 2-bromobenzamide (1.00 g, 5.00 $\mathrm{mmol}), \mathrm{Pd}(\mathrm{OAc})_{2}(0.056 \mathrm{~g}, 0.25 \mathrm{mmol})$, CuI (0.048 g, $0.25 \mathrm{mmol}), \mathrm{PPh}_{3}(0.13 \mathrm{~g}, 0.50 \mathrm{mmol})$, 1-hexyne $(0.62 \mathrm{~g}, 0.86 \mathrm{~mL}, 7.50 \mathrm{mmol})$ and $\mathrm{Et}_{3} \mathrm{~N}(40 \mathrm{~mL})$. Upon completion (24 h) and work-up, the residue was purified by flash column chromatography through silica gel using a 1:1 mixture of EtOAc/Hex as eluent to afford $3 \mathbf{e}(0.18 \mathrm{~g}, 18 \%)$ as a brown solid.

${ }^{1} \mathrm{H}$ NMR (250 MHz, $\left.\mathrm{CDCl}_{3}\right): \delta=8.18-8.01(\mathrm{~m}, 1 \mathrm{H})$, 7.77-7.31 (m, 5H), 2.46 (t, $J=7.0 \mathrm{~Hz}, 2 \mathrm{H}), 1.68-1.40$ (m, 4H), 0.94 (t, $J=7.1 \mathrm{~Hz}, 3 \mathrm{H})$.

${ }^{13} \mathrm{C}$ NMR, DEPT $\left(62 \mathrm{MHz}, \mathrm{CDCl}_{3}\right): \delta=168.7(\mathrm{C}=\mathrm{O})$, 134.1 (C), 133.5 (CH), $130.6(\mathrm{CH}), 129.8(\mathrm{CH}), 127.8$ (CH), $120.8(\mathrm{C}), 97.6(\mathrm{C}), 79.3(\mathrm{C}), 30.2\left(\mathrm{CH}_{2}\right), 21.8$ $\left(\mathrm{CH}_{2}\right), 19.1\left(\mathrm{CH}_{2}\right), 13.3\left(\mathrm{CH}_{3}\right)$.

MS (CI): m/z (\%) = $202\left(\mathrm{M}^{+}+1,100\right), 186$ (12).

\section{$N$-(2-ethynylbenzyl)acetamide (3f)}

Acetic anhydride (1.15 g, $11.29 \mathrm{mmol})$ was added dropwise to a solution of (2bromophenyl)methanamine (2.00 g, $10.75 \mathrm{mmol})$ in $\mathrm{Et}_{3} \mathrm{~N}(40 \mathrm{~mL})$ at room temperature. The reaction mixture was stirred for $12 \mathrm{~h}$ until disappearance of starting material (TLC, GC-MS monitoring). Excess of acetic anhydride was quenched with $\mathrm{H}_{2} \mathrm{O}(40 \mathrm{~mL})$ and the mixture was extracted with EtOAc ( 3 x 10 $\mathrm{mL})$. The combined organic layers were dried over anhydrous $\mathrm{Na}_{2} \mathrm{SO}_{4}$, filtered and evaporated under vacuum to afford quantitatively $N$-(2bromobenzyl)acetamide (2.44 g) as a white solid. mp: 81-82.5 ${ }^{\circ} \mathrm{C}$

${ }^{1} \mathrm{H}$ NMR $\left(250 \mathrm{MHz}, \mathrm{CDCl}_{3}\right): \delta=7.51(\mathrm{~d}, J=7.9 \mathrm{~Hz}$, 1H), 7.34-7.21 (m, 2H), 7.11 (t, $J=7.5 \mathrm{~Hz}, 1 \mathrm{H}), 6.62$ (s, 1H), 4.43 (d, $J=6.0 \mathrm{~Hz}, 2 \mathrm{H}), 1.99$ (s, 3H).

${ }^{13} \mathrm{C}$ NMR, DEPT $\left(62 \mathrm{MHz}, \mathrm{CDCl}_{3}\right)$ : $\delta=170.2(\mathrm{C}=\mathrm{O})$, 137.1 (C), 132.6 (CH), $129.8(\mathrm{CH}), 128.9(\mathrm{CH}), 127.5$ $(\mathrm{CH}), 123.4(\mathrm{C}), 43.6\left(\mathrm{CH}_{2}\right), 22.9\left(\mathrm{CH}_{3}\right)$.

MS (CI): m/z (\%) = $228\left(\mathrm{M}^{+}+1,100\right), 171$ (15), 148 (14).

In a flame-dried round-bottomed flask under Ar was suspended $N$-(2-bromobenzyl)acetamide $(2.37 \mathrm{~g}$, $10.44 \mathrm{mmol}), \mathrm{PdCl}_{2}(0.28 \mathrm{~g}, 1.57), \mathrm{Cu}(\mathrm{OAc})_{2}(0.38 \mathrm{~g}$, $2.09 \mathrm{mmol}), \mathrm{PPh}_{3}(1.37 \mathrm{~g}, 5.22 \mathrm{mmol})$ in $\mathrm{Et}_{3} \mathrm{~N}$ (100 $\mathrm{mL}$ ) and trimethylsilylacetylene (2.95 g, $2.95 \mathrm{~mL}$, $20.88 \mathrm{mmol}$ ). The reaction mixture was stirred at 90 ${ }^{\circ} \mathrm{C}$ for $17 \mathrm{~h}$ until disappearance of starting material (TLC, GC-MS monitoring). The reaction mixture was filtered through silica gel using EtOAc as eluent. The filtrate was evaporated under vacuum and the residue was purified by flash column chromatography through silica gel using a 1:1 mixture of EtOAc/Hex as eluent to give $N-\{2-$ [(trimethylsilyl)ethynyl)benzyl \}acetamide (1.10 $43 \%)$ as a brown solid.

${ }^{1} \mathrm{H}$ NMR (250 MHz, $\left.\mathrm{CDCl}_{3}\right): \delta=7.45(\mathrm{~d}, J=6.7 \mathrm{~Hz}$, 1H), 7.31-7.18 (m, 3H), 6.35 (s, 1H), 4.54 (d, $J=5.9$ $\mathrm{Hz}, 2 \mathrm{H}), 1.98$ (s, 3H), 0.27 (s, 9H).

${ }^{13} \mathrm{C}$ NMR, DEPT (62 MHz, $\left.\mathrm{CDCl}_{3}\right): \delta=169.9(\mathrm{C}=\mathrm{O})$, $140.1(\mathrm{C}), 132.4(\mathrm{CH}), 128.8(\mathrm{CH}), 128.1(\mathrm{CH}), 127.1$ (CH), $122.0(\mathrm{C}), 102.7(\mathrm{C}), 99.3(\mathrm{C}), 42.2\left(\mathrm{CH}_{2}\right), 23.0$ $\left(\mathrm{CH}_{3}\right),-0.2\left(3 \mathrm{x} \mathrm{CH}_{3}\right)$.

MS (CI): m/z (\%) = $246\left(\mathrm{M}^{+}+1,100\right), 230$ (33), 204 (31).

The general desilylation procedure was followed using $N$-\{2-[(trimethylsilyl)ethynyl]benzyl $\}$ acetamide $(0.22 \mathrm{~g}, 1.63 \mathrm{mmol})$, a solution of TBAF in THF (1M, $1.63 \mathrm{~mL})$ and THF (15 mL). Upon completion (10 min) and work-up, the residue was purified by flash column chromatography through silica gel using a 7:3 mixture of EtOAc/Hex as eluent to give $3 \mathbf{f}(0.21 \mathrm{~g}$, $75 \%)$ as a yellowish solid.

${ }^{1} \mathrm{H}$ NMR $\left(250 \mathrm{MHz}, \mathrm{CDCl}_{3}\right): \delta=7.46(\mathrm{~d}, J=7.1 \mathrm{~Hz}$, 1H), 7.33-7.16 (m, 3H), 6.71 (s, 1H), 4.52 (d, $J=5.9$ $\mathrm{Hz}, 2 \mathrm{H}), 3.33$ (s, 1H), 1.94 (s, 3H).

${ }^{13} \mathrm{C}$ NMR, DEPT (62 MHz, $\left.\mathrm{CDCl}_{3}\right): \delta=170.1(\mathrm{C}=\mathrm{O})$, $140.5(\mathrm{C}), 132.6(\mathrm{CH}), 129.0(\mathrm{CH}), 128.0(\mathrm{CH}), 127.0$ $(\mathrm{CH}), 120.8(\mathrm{C}), 82.0(\mathrm{CH}), 81.2(\mathrm{C}), 41.8\left(\mathrm{CH}_{2}\right)$, $22.8\left(\mathrm{CH}_{3}\right)$.

MS (CI): m/z (\%) = $174\left(\mathrm{M}^{+}+1,71\right), 160$ (11), 132 (100).

\section{$N$-(2-ethynylbenzyl)-4-methylbenzenesulfonamide (3g)}

A solution of $p$-toluenesulfonyl chloride (2.15 g, 11.30 $\mathrm{mL})$ in $\mathrm{Et}_{3} \mathrm{~N}(4.50 \mathrm{~mL})$ was cannulated to a solution of (2-bromophenyl)methanamine $(2.00 \mathrm{~g}, \quad 10.76$ mmol) in $\mathrm{CH}_{2} \mathrm{Cl}_{2}(22 \mathrm{~mL})$ cooled at $0^{\circ} \mathrm{C}$. The reaction mixture was stirred for $12 \mathrm{~h}$ at room temperature until disappearance of starting material (TLC, GC-MS monitoring). The reaction was quenched adding $\mathrm{H}_{2} \mathrm{O}$ $(20 \mathrm{~mL})$ and then extracted with EtOAc $(3 \times 10 \mathrm{~mL})$. The combined organic layers were dried over anhydrous $\mathrm{Na}_{2} \mathrm{SO}_{4}$, filtered and evaporated under vacuum. The residue was purified by flash column chromatography through silica gel using a 1:4 mixture of EtOAc/Hex as eluent to give $N$-(2-bromobenzyl)-4methylbenzenesulfonamide $(2.85 \mathrm{~g}, 75 \%)$ as a white solid.

${ }^{1} \mathrm{H}$ NMR (500 MHz, $\left.\mathrm{CDCl}_{3}\right): \delta=7.71(\mathrm{~d}, J=8.2 \mathrm{~Hz}$, 2H), 7.43 (d, $J=7.8 \mathrm{~Hz}, 1 \mathrm{H}), 7.30$ (d, $J=7.5 \mathrm{~Hz}$, $1 \mathrm{H}), 7.23$ (d, $J=8.2 \mathrm{~Hz}, 2 \mathrm{H}), 7.19(\mathrm{t}, J=7.5 \mathrm{~Hz}, 1 \mathrm{H})$, 7.09 (t, $J=7.8 \mathrm{~Hz}, 1 \mathrm{H}), 5.20$ (t, $J=6.5 \mathrm{~Hz}, 1 \mathrm{H}), 4.21$ (d, $J=6.5 \mathrm{~Hz}, 2 \mathrm{H}), 2.39$ (s, 3H).

${ }^{13} \mathrm{C}$ NMR, DEPT (125 MHz, $\left.\mathrm{CDCl}_{3}\right): \delta=143.4(\mathrm{C})$, 136.8 (C), 135.5 (C), $132.6(\mathrm{CH}), 130.3(\mathrm{CH}), 129.5$ (2 x CH), $129.4(\mathrm{CH}), 127.6(\mathrm{CH}), 127.0(2 \times \mathrm{CH})$, $123.3(\mathrm{C}), 47.3\left(\mathrm{CH}_{2}\right), 21.4\left(\mathrm{CH}_{3}\right)$.

MS (CI): m/z (\%) = $340\left(\mathrm{M}^{+}+1,8\right), 260$ (68), 184 (100), 155 (23), 139 (19), 91 (77), 77 (25), 65 (22). 
HRMS-EI: $\mathrm{m} / \mathrm{z}$ calcd for $\mathrm{C}_{14} \mathrm{H}_{14} \mathrm{NO}_{2} \mathrm{SBr} \quad\left[\mathrm{M}^{+}\right]$: 338.9929; found: 338.9914.

The general Sonogashira cross coupling procedure 2 was followed using $N$-(2-bromobenzyl)-4methylbenzenesulfonamide (1.44 g, $4.25 \mathrm{mmol})$, $\mathrm{Pd}(\mathrm{OAc})_{2}(0.029 \mathrm{~g}, 0.13 \mathrm{mmol})$, CuI (0.024 g, 0.13 $\mathrm{mmol}), \quad \mathrm{PPh}_{3} \quad(0.056 \quad \mathrm{~g}, \quad 0.21 \mathrm{mmol})$, trimethylsilylacetylene $(0.63 \mathrm{~g}, 0.90 \mathrm{~mL}, 3.38 \mathrm{mmol})$ and $\mathrm{Et}_{3} \mathrm{~N}(40 \mathrm{~mL})$. Upon completion $(8 \mathrm{~h})$ and workup, the residue was purified by flash column chromatography through silica gel using a 1:4 mixture of EtOAc/Hex as eluent to afford 4-methyl- $N$ - $\{2$ [(trimethylsilyl)ethynyl]benzyl $\}$ benzenesulfonamide (0.56 g, 37\%) as a brown solid. To this solid solved in THF $(15 \mathrm{~mL})$ was added a solution of TBAF in THF $(1 \mathrm{M}, 2.34 \mathrm{~mL})$ and the resulting solution stirred at room temperature. Upon completion (10 min) and work-up, the residue was purified by flash column chromatography through silica gel using a 1:4 mixture of EtOAc/Hex as eluent to give $\mathbf{3 g}(0.28 \mathrm{~g}, 63 \%)$ as a brown solid.

${ }^{1} \mathrm{H}$ NMR (500 MHz, $\left.\mathrm{CDCl}_{3}\right): \delta=7.70(\mathrm{~d}, J=8.2 \mathrm{~Hz}$, 2H), 7.40 (d, $J=7.3 \mathrm{~Hz}, 1 \mathrm{H}$ ), 7.25-7.18 (m, 5H), 5.02 (t, $J=6.2 \mathrm{~Hz}, 1 \mathrm{H}$ ), 4.30 (d, $J=6.2 \mathrm{~Hz}, 2 \mathrm{H}$ ), 3.20 (s, $1 \mathrm{H}), 2.40$ (s, 3H).

${ }^{13} \mathrm{C}$ NMR, DEPT (125 MHz, $\left.\mathrm{CDCl}_{3}\right): \delta=143.3(\mathrm{C})$, 138.6 (C), 137.0 (C), $132.9(\mathrm{CH}), 129.5(2 \times \mathrm{CH})$, 129.2 (CH), $128.8(\mathrm{CH}), 127.7(\mathrm{CH}), 127.1(2 \times \mathrm{CH})$, 121.0 (C), $82.4(\mathrm{CH}), 81.0(\mathrm{C}), 46.0\left(\mathrm{CH}_{2}\right), 21.3$ $\left(\mathrm{CH}_{3}\right)$.

MS (CI): $\mathrm{m} / \mathrm{z}(\%)=286\left(\mathrm{M}^{+}+1,100\right)$.

HRMS-EI: $\mathrm{m} / \mathrm{z}$ calcd for $\mathrm{C}_{16} \mathrm{H}_{15} \mathrm{NO}_{2} \mathrm{~S} \quad\left[\mathrm{M}^{+}\right]$: 285.0823; found: 285.0828 .

\section{4-Methyl- $N$-(2-(prop-2-yn-1- yl)phenyl)benzenesulfonamide (5)}

To a solution of (2-aminophenyl)methanol (1.00 g, $8.12 \mathrm{mmol})$ in $\mathrm{CH}_{2} \mathrm{Cl}_{2}(50 \mathrm{~mL})$ was added $\mathrm{MnO}_{2}$ $(0.70 \mathrm{~g}, 8.12 \mathrm{mmol})$ at room temperature. The reaction mixture was stirred for $24 \mathrm{~h}$ until disappearance of starting material (TLC, GC-MS monitoring). The mixture was filtered through silica gel using EtOAc as eluent. The filtrate was evaporated under vacuum and the residue was purified by flash column chromatography through silica gel using a 3:7 mixture of EtOAc/Hex as eluent to give 2-aminobenzaldehyde (0.49g, $50 \%)$ as a yellow oil.

${ }^{1} \mathrm{H}$ NMR (250 MHz, $\left.\mathrm{CDCl}_{3}\right): \delta=9.88$ (s, $\left.1 \mathrm{H}\right), 7.49$ (dd, $J=7.8,1.4 \mathrm{~Hz}, 1 \mathrm{H}$ ), 7.36-7.28 (m, 1H), 6.75 (t, $J$ $=7.5 \mathrm{~Hz}, 1 \mathrm{H}), 6.65(\mathrm{~d}, J=8.3 \mathrm{~Hz}, 1 \mathrm{H}), 6.10(\mathrm{~s}, 2 \mathrm{H})$.

MS (CI): m/z (\%) = $122\left(\mathrm{M}^{+}+1,100\right), 94$ (6).

A solution of $p$-toluenesulfonyl chloride $(0.70 \mathrm{~g}, 3.64$ mmol) in $\mathrm{CH}_{2} \mathrm{Cl}_{2}(7 \mathrm{~mL})$ was cannulated to a solution of 2-aminobenzaldehyde $(0.40 \mathrm{~g}, 3.31 \mathrm{mmol})$ and pyridine (0.58 g, $0.60 \mathrm{~mL}, 7.28 \mathrm{mmol})$ in $\mathrm{CH}_{2} \mathrm{Cl}_{2}(10$ $\mathrm{mL}$ ) cooled at $0{ }^{\circ} \mathrm{C}$. The reaction mixture was stirred for $12 \mathrm{~h}$ at room temperature until disappearance of starting material (TLC, GC-MS monitoring). The reaction was quenched adding $\mathrm{H}_{2} \mathrm{O}(10 \mathrm{~mL})$ and then was extracted with EtOAc ( 3 x $5 \mathrm{~mL}$ ). The combined organic layers were dried over anhydrous $\mathrm{Na}_{2} \mathrm{SO}_{4}$, filtered and evaporated under vacuum. The residue was purified by flash column chromatography through silica gel using 1:4 mixture of EtOAc/Hex as eluent to give $\quad \mathrm{N}$-(2-formylphenyl)-4methylbenzenesulfonamide (0.83 $\mathrm{g}, 91 \%$ ) as a brown oil.

${ }^{1} \mathrm{H}$ NMR (250 MHz, $\left.\mathrm{CDCl}_{3}\right): \delta=10.79(\mathrm{~s}, 1 \mathrm{H}), 9.83$ (s, $1 \mathrm{H}), 7.78(\mathrm{~d}, J=8.4 \mathrm{~Hz}, 2 \mathrm{H}), 7.69(\mathrm{~d}, J=8.4 \mathrm{~Hz}$, $1 \mathrm{H}), 7.62-7.57(\mathrm{~m}, 1 \mathrm{H}), 7.55-7.47(\mathrm{~m}, 1 \mathrm{H}), 7.27-7.22$ (m, 2H), 7.19-7.11 (m, 1H), 2.37 (s, 3H).

MS (CI): m/z (\%) = $276\left(\mathrm{M}^{+}+1,50\right), 248$ (20), 155 (26), 125 (100).

A $5 \mathrm{M}$ solution of ethynyl magnesium bromide in THF (5.60 mL, $2.80 \mathrm{mmol}$ ) was added dropwise to a solution of $\quad \mathrm{N}$-(2-formylphenyl)-4methylbenzenesulfonamide $(0.70 \mathrm{~g}, 2.54 \mathrm{mmol})$ in THF $(25 \mathrm{~mL})$ cooled at $0{ }^{\circ} \mathrm{C}$. The reaction mixture was stirred for $7 \mathrm{~h}$ at $0{ }^{\circ} \mathrm{C}$ until disappearance of starting material(TLC, GC-MS monitoring). The mixture was evaporated under vacuum and the residue was dissolved in EtOAc $(20 \mathrm{~mL})$, washed with brine (2 x $10 \mathrm{~mL}$ ), dried over anhydrous $\mathrm{Na}_{2} \mathrm{SO}_{4}$, filtered and evaporated under vacuum. The residue was purified by flash column chromatography through silica gel using a 1:1 mixture of EtOAc/Hex as eluent to give $N$-[2-(1-hydroxyprop-2-yn-1-yl)phenyl]-4methylbenzenesulfonamide $(0.72 \mathrm{~g}, 94 \%)$ as a yellowish oil.

${ }^{1} \mathrm{H}$ NMR (250 MHz, $\left.\mathrm{CDCl}_{3}\right): \delta=7.97(\mathrm{~s}, 1 \mathrm{H}), 7.65(\mathrm{~d}$, $J=8.2 \mathrm{~Hz}, 2 \mathrm{H}), 7.51(\mathrm{~d}, J=7.6 \mathrm{~Hz}, 1 \mathrm{H}), 7.33-7.09$ (m, 5H), 5.34 (d, $J=2.0 \mathrm{~Hz}, 1 \mathrm{H}), 3.69(\mathrm{~s}, 1 \mathrm{H}), 2.64$ (d, $J=2.2 \mathrm{~Hz}, 1 \mathrm{H}$ ), 2.36 (s, 3H).

${ }^{13} \mathrm{C}$ NMR, DEPT (62 MHz, $\left.\mathrm{CDCl}_{3}\right): \delta=143.9(\mathrm{C})$, 136.3 (C), 135.0 (C), 131.2 (C), 129.6 (2 x CH), 129.5 $(\mathrm{CH}), 128.7(\mathrm{CH}), 127.1(2 \times \mathrm{CH}), 125.5(\mathrm{CH}), 123.2$ (CH), $81.4(\mathrm{C}), 76.3(\mathrm{CH}), 62.2(\mathrm{CH}), 21.4\left(\mathrm{CH}_{3}\right)$.

A solution of $\mathrm{Et}_{3} \mathrm{SiH}(0.50 \mathrm{~g}, 0.69 \mathrm{~mL}, 4.32 \mathrm{mmol})$ in TFA ( $1 \mathrm{~g}, 0.67 \mathrm{~mL}, 8.64 \mathrm{mmol})$ was slowly added to a solution of $N$-[2-(1-hydroxyprop-2-yn-1-yl)phenyl]-4methylbenzenesulfonamide $(0.65 \mathrm{~g}, 2.16 \mathrm{mmol})$ in THF $(20 \mathrm{~mL})$. The reaction mixture was stirred for 12 $\mathrm{h}$ at room temperature until disappearance of starting material (TLC, GC-MS monitoring). The reaction was quenched by adding a saturated solution of $\mathrm{NaHCO}_{3}$ $(15 \mathrm{~mL})$ and extracted with $\mathrm{CH}_{2} \mathrm{Cl}_{2}(3 \times 10 \mathrm{~mL})$. The combined organic layers were dried over anhydrous $\mathrm{Na}_{2} \mathrm{SO}_{4}$, filtered and evaporated under vacuum. The residue was purified by flash column chromatography through silica gel using a 3:7 mixture of EtOAc/Hex as eluent to afford $4(0.32 \mathrm{~g}, 51 \%)$ as a brown oil.

${ }^{1} \mathrm{H}$ NMR $\left(250 \mathrm{MHz}, \mathrm{CDCl}_{3}\right): \delta=7.61(\mathrm{~d}, J=8.2 \mathrm{~Hz}$, $2 \mathrm{H}), 7.27-7.15$ (m, 6H), 6.99 (s, 1H), 3.24 (d, $J=2.6$ Hz, 2H), 2.39 (s, 3H), 2.04 (s, 1H).

${ }^{13} \mathrm{C}$ NMR, DEPT (62 MHz, $\mathrm{CDCl}_{3}$ ): $\delta=143.8$ (C), 136.6 (C), 134.2 (C), 130.7 (C), 129.6 (2 x CH), 129.5 
(CH), $128.0(\mathrm{CH}), 127.0$ (2 x CH), $126.8(\mathrm{CH}), 125.9$ (CH), $80.4(\mathrm{C}), 71.8(\mathrm{CH}), 21.6\left(\mathrm{CH}_{2}\right), 21.5\left(\mathrm{CH}_{3}\right)$.

MS (CI): m/z (\%) = $286\left(\mathrm{M}^{+}+1,100\right), 160$ (30), 132 (94).

\section{General procedure for the Ru-catalyzed heterocyclization reactions}

Aromatic alkynyl amines/amides (1 equiv) were added to a suspension of the ruthenium catalyst (10 mol\%) in pyridine $(0.15 \mathrm{M})$ in a flame-dried sealed tube under $\mathrm{Ar}$ and the reaction mixture was heated at $90{ }^{\circ} \mathrm{C}$ until the complete consumption of the starting material (GC-MS monitoring). The reaction mixture was then cooled at room temperature, washed with saturated aqueous solution of $\mathrm{NH}_{4} \mathrm{Cl}$ and extracted with diethyl ether $(3 \mathrm{x})$. The combined organic layers were dried over anhydrous $\mathrm{Na}_{2} \mathrm{SO}_{4}$, filtered and evaporated under vacuum. The residue was purified by flash column chromatography through silica gel using a mixture of EtOAc/Hex as eluent to afford the corresponding heterocyclization product.

\section{H-indole (2a)}

The general heterocyclization procedure was followed using 1a (0.058 g, $0.50 \mathrm{mmol}), \mathrm{CpRuCl}\left(\mathrm{PPh}_{3}\right)_{2}(0.036$ g, $0.050 \mathrm{mmol})$ and pyridine $(3.30 \mathrm{~mL})$. Upon completion (25 min), the reaction was worked-up and the residue was purified by flash column chromatography through silica gel using a 1:4 mixture of EtOAc/Hex as eluent to give 2a (0.049 g, $84 \%)$ as a yellow solid.

${ }^{1} \mathrm{H}$ NMR (300 MHz, $\mathrm{CDCl}_{3}$ ), $\delta(\mathrm{ppm}): 8.02$ (s, 1H), $7.65(\mathrm{~d}, J=7.8 \mathrm{~Hz}, 1 \mathrm{H}), 7.34(\mathrm{~d}, J=7.8 \mathrm{~Hz}, 1 \mathrm{H})$, 7.21-7.09 (m, 3H), 6.54 (d, $J=2.0 \mathrm{~Hz}, 1 \mathrm{H})$.

${ }^{13} \mathrm{C}$ NMR, DEPT (75 MHz, $\mathrm{CDCl}_{3}$ ), $\delta$ (ppm): 135.7 (C), $127.8(\mathrm{C}), 124.1(\mathrm{CH}), 121.9(\mathrm{CH}), 120.7(\mathrm{CH})$, $119.8(\mathrm{CH}), 110.9(\mathrm{CH}), 102.5(\mathrm{CH})$.

MS (CI): m/z (\%) = $118\left(\mathrm{M}^{+}+1,100\right), 91$ (14), 77 (15).

\section{1-(Methylsulfonyl)-1H-indole (2b)}

The general heterocyclization procedure was followed using $1 \mathbf{b}(0.098 \mathrm{~g}, 0.50 \mathrm{mmol})$, $\mathrm{CpRuCl}\left(\mathrm{PPh}_{3}\right)_{2}(0.036$ g, $0.050 \mathrm{mmol})$ and pyridine $(3.30 \mathrm{~mL})$. Upon completion (40 min) and work-up, the residue was purified by flash column chromatography through silica gel using a 1:9 mixture of EtOAc/Hex as eluent to give $\mathbf{2 b}(0.072 \mathrm{~g}, 73 \%)$ as a yellowish oil.

${ }^{1} \mathrm{H}$ NMR (250 MHz, $\mathrm{CDCl}_{3}$ ), $\delta$ (ppm): 7.91 (d, $J=8.3$ $\mathrm{Hz}, 1 \mathrm{H}$ ), 7.62 (dd, $J=6.9,1.4 \mathrm{~Hz}, 1 \mathrm{H}), 7.43$ (d, $J=$ $3.7 \mathrm{~Hz}, 1 \mathrm{H}), 7.39-7.24(\mathrm{~m}, 2 \mathrm{H}), 6.71(\mathrm{~d}, J=3.7 \mathrm{~Hz}$, $1 \mathrm{H}), 3.08$ (s, 3H).

${ }^{13} \mathrm{C}$ NMR, DEPT (75 MHz, $\left.\mathrm{CDCl}_{3}\right), \delta(\mathrm{ppm}): 134.8$ (C), $130.6(\mathrm{C}), 126.1(\mathrm{CH}), 124.8(\mathrm{CH}), 123.5(\mathrm{CH})$, 121.6 (CH), $112.9(\mathrm{CH}), 108.8(\mathrm{CH}), 40.6\left(\mathrm{CH}_{3}\right)$.

MS (EI, $70 \mathrm{eV}): \mathrm{m} / \mathrm{z}(\%)=195\left(\mathrm{M}^{+}, 24\right), 116(100)$, 89 (43), 63 (28).

HRMS-EI: $\mathrm{m} / \mathrm{z}$ calcd for $\mathrm{C}_{9} \mathrm{H}_{9} \mathrm{NO}_{2} \mathrm{~S}\left[\mathrm{M}^{+}\right]$: 195.0354; found: 195.0354 .

\section{1-Tosyl-1H-indole (2c)}

The general heterocyclization procedure was followed using 1c (0.136 g, $0.50 \mathrm{mmol}), \mathrm{CpRuCl}\left(\mathrm{PPh}_{3}\right)_{2}(0.036$ g, $0.050 \mathrm{mmol})$ and pyridine $(3.30 \mathrm{~mL})$. Upon completion (40 min) and work-up, the residue was purified by flash column chromatography through silica gel using a 1:4 mixture of EtOAc/Hex as eluent to give $2 \mathrm{c}(0.108 \mathrm{~g}, 80 \%)$ as a yellowish oil.

${ }^{1} \mathrm{H}$ NMR (250 MHz, $\mathrm{CDCl}_{3}$ ), $\delta$ (ppm): 7.99 (d, $J=8.1$ $\mathrm{Hz}, 1 \mathrm{H}), 7.76$ (d, $J=8.4 \mathrm{~Hz}, 2 \mathrm{H}), 7.59-7.49$ (m, 2H), 7.33-7.18 (m, 4H), 6.65 (d, $J=3.7 \mathrm{~Hz}, 1 \mathrm{H}), 2.32$ (s, $3 \mathrm{H})$.

${ }^{13} \mathrm{C}$ NMR, DEPT (75 MHz, $\left.\mathrm{CDCl}_{3}\right), \delta$ (ppm): 144.9 (C), 135.3 (C), 134.8 (C), 130.7 (C), 129.8 (2 x CH), $126.8(2 \times \mathrm{CH}), 126.4(\mathrm{CH}), 124.5(\mathrm{CH}), 123.2(\mathrm{CH})$, $121.3(\mathrm{CH}), 113.6(\mathrm{CH}), 109.0(\mathrm{CH}), 21.5\left(\mathrm{CH}_{3}\right)$.

MS (CI): m/z (\%) = $272\left(\mathrm{M}^{+}+1,8\right), 271$ (72), 180 (53), 91 (100).

HRMS-CI: $\mathrm{m} / \mathrm{z}$ calcd for $\mathrm{C}_{15} \mathrm{H}_{14} \mathrm{NO}_{2} \mathrm{~S} \quad\left[\mathrm{M}^{+}+1\right]$ : 272.0745; found: 272.0745 .

\section{5-Nitro-1H-indole (2d)}

The general heterocyclization procedure was followed using $1 \mathrm{~d}(0.081 \mathrm{~g}, 0.50 \mathrm{mmol}), \mathrm{CpRuCl}\left(\mathrm{PPh}_{3}\right)_{2}(0.036$ g, $0.050 \mathrm{mmol})$ and pyridine $(3.30 \mathrm{~mL})$. Upon completion (20 $\mathrm{min}$ ) and work-up, the residue was purified by flash column chromatography through silica gel using a 1:4 mixture of EtOAc/Hex as eluent to give $2 \mathbf{d}(0.058 \mathrm{~g}, 72 \%)$ as a yellow solid.

${ }^{1} \mathrm{H}$ NMR (300 MHz, $\left.\mathrm{CDCl}_{3}\right), \delta(\mathrm{ppm}): 8.71$ (s, $\left.1 \mathrm{H}\right)$, 8.62 (s, $1 \mathrm{H}), 8.12$ (d, $J=9.0 \mathrm{~Hz}, 1 \mathrm{H}), 7.45$ (d, $J=9.0$ $\mathrm{Hz}, 1 \mathrm{H}), 7.39$ (bs, 1H), 6.74 (s, 1H).

${ }^{13} \mathrm{C}$ NMR, DEPT (75 MHz, $\left.\mathrm{CDCl}_{3}\right), \delta$ (ppm): 141.9 (C), $138.8(\mathrm{C}), 127.4(\mathrm{CH}), 127.2(\mathrm{C}), 118.0(\mathrm{CH})$, 117.6 (CH), $111.0(\mathrm{CH}), 105.0(\mathrm{CH})$.

MS (EI, $70 \mathrm{eV}): \mathrm{m} / \mathrm{z}(\%)=162\left(\mathrm{M}^{+}, 100\right), 132(15)$, 116 (95), 104 (22), 89 (70), 63 (35).

HRMS-EI: $\mathrm{m} / \mathrm{z}$ calcd for $\mathrm{C}_{8} \mathrm{H}_{6} \mathrm{~N}_{2} \mathrm{O}_{2}\left[\mathrm{M}^{+}\right]:$: 162.0429; found: 162.0429 .

\section{$1 H$-indole-5-carbonitrile (2e)}

The general heterocyclization procedure was followed using 1e $(0.071 \mathrm{~g}, 0.50 \mathrm{mmol}), \mathrm{CpRuCl}\left(\mathrm{PPh}_{3}\right)_{2}(0.036$ g, $\quad 0.050 \mathrm{mmol})$ and pyridine $(3.30 \mathrm{~mL})$. Upon completion (30 min) and work-up, the residue was purified by flash column chromatography through silica gel using a 1:4 mixture of EtOAc/Hex as eluent to give $2 \mathbf{e}(0.070 \mathrm{~g}, 98 \%)$ as a yellow solid.

${ }^{1} \mathrm{H}$ NMR (300 MHz, $\left.\mathrm{CDCl}_{3}\right), \delta(\mathrm{ppm}): 9.10(\mathrm{~s}, 1 \mathrm{H})$, 7.98 (s, $1 \mathrm{H}), 7.47$ (d, $J=8.5 \mathrm{~Hz}, 1 \mathrm{H}), 7.39$ (dd, $J=$ 8.5, 1.4 Hz, 1H), 7.34-7.32 (m, 1H), 6.60 (bs, 1H).

${ }^{13} \mathrm{C}$ NMR, DEPT (75 MHz, $\left.\mathrm{CDCl}_{3}\right), \delta(\mathrm{ppm}): 137.5$ (C), $128.9(\mathrm{C}), 126.6(\mathrm{CH}), 126.2(\mathrm{CH}), 124.6(\mathrm{CH})$, $121.0(\mathrm{CN}), 112.1(\mathrm{CH}), 103.1(\mathrm{CH}), 102.3(\mathrm{C})$.

MS (EI, $70 \mathrm{eV}): \mathrm{m} / \mathrm{z}(\%)=142\left(\mathrm{M}^{+}, 100\right), 115(36)$, $88(10)$. 
HRMS-EI: $\mathrm{m} / \mathrm{z}$ calcd for $\mathrm{C}_{9} \mathrm{H}_{6} \mathrm{~N}_{2}\left[\mathrm{M}^{+}\right]$: 142.0531; found: 142.0531 .

\section{Methyl $1 \mathrm{H}$-indole-5-carboxylate (2f)}

The general heterocyclization procedure was followed using 1 f $(0.088 \mathrm{~g}, 0.50 \mathrm{mmol})$, $\mathrm{CpRuCl}\left(\mathrm{PPh}_{3}\right)_{2}(0.036$ g, $0.050 \mathrm{mmol})$ and pyridine $(3.30 \mathrm{~mL})$. Upon completion (30 min) and work-up, the residue was purified by flash column chromatography through silica gel using a 1:4 mixture of EtOAc/Hex as eluent to give $2 \mathbf{f}(0.086 \mathrm{~g}, 98 \%)$ as a yellow solid.

${ }^{1} \mathrm{H}$ NMR (300 MHz, $\mathrm{CDCl}_{3}$ ), $\delta$ (ppm): 8.85 (s, 1H), 8.43 (s, 1H), 7.89 (dd, $J=8.6,1.5 \mathrm{~Hz}, 1 \mathrm{H}), 7.37$ (d, $J$ = $8.6 \mathrm{~Hz}, 1 \mathrm{H}), 7.23-7.21$ (m, 1H), 6.61 (bs, $1 \mathrm{H}), 3.92$ (s, 3H).

${ }^{13} \mathrm{C}$ NMR, DEPT (75 MHz, $\left.\mathrm{CDCl}_{3}\right), \delta$ (ppm): 168.4 $(\mathrm{C}=\mathrm{O}), 138.4(\mathrm{C}), 128.8(\mathrm{C}), 125.9(\mathrm{CH}), 123.7(\mathrm{CH})$, $123.1(\mathrm{CH}), 121.6(\mathrm{C}), 110.8(\mathrm{CH}), 103.7(\mathrm{CH}), 51.8$ $\left(\mathrm{CH}_{3}\right)$.

MS (EI, $70 \mathrm{eV}): \mathrm{m} / \mathrm{z}(\%)=175\left(\mathrm{M}^{+}, 67\right), 144(100)$, 116 (55), 89 (21), 58 (44).

HRMS-EI: $\mathrm{m} / \mathrm{z}$ calcd for $\mathrm{C}_{10} \mathrm{H}_{9} \mathrm{NO}_{2}\left[\mathrm{M}^{+}\right]$: 175.0633; found: 175.0633 .

\section{Methyl 1H-indole-6-carboxylate (2g)}

The general heterocyclization procedure was followed using $1 \mathrm{~g}$ (0.088 g, $0.50 \mathrm{mmol})$, $\mathrm{CpRuCl}\left(\mathrm{PPh}_{3}\right)_{2}(0.036$ g, $0.050 \mathrm{mmol})$ and pyridine $(3.30 \mathrm{~mL})$. Upon completion (90 min) and work-up, the residue was purified by flash column chromatography through silica gel using a 1:4 mixture of EtOAc/Hex as eluent to give $2 \mathbf{g}(0.081 \mathrm{~g}, 92 \%)$ as a yellow solid.

${ }^{1} \mathrm{H}$ NMR (250 MHz, $\mathrm{CDCl}_{3}$ ), $\delta$ (ppm): 8.70 (bs, $1 \mathrm{H}$ ), 8.16 (bs, $1 \mathrm{H}$ ), 7.82 (dd, $J=8.4,1.4 \mathrm{~Hz}, 1 \mathrm{H}$ ), 7.62 (d, $J=8.4 \mathrm{~Hz}, 1 \mathrm{H}), 7.32(\mathrm{t}, J=2.8 \mathrm{~Hz} 1 \mathrm{H}), 6.57(\mathrm{~m}$, $1 \mathrm{H}), 3.92(\mathrm{~s}, 3 \mathrm{H})$.

\section{Isoquinolin-1(2H)-one (4b)}

The general heterocyclization procedure was followed using $3 \mathbf{b}(0.072 \mathrm{~g}, 0.50 \mathrm{mmol})$, $\mathrm{CpRuCl}\left(\mathrm{PPh}_{3}\right)_{2}(0.036$ g, $0.050 \mathrm{mmol})$ and pyridine $(3.30 \mathrm{~mL})$. Upon completion (60 $\mathrm{min}$ ) and work-up, the residue was purified by flash column chromatography through silica gel using a 1:1 mixture of EtOAc/Hex as eluent to give $\mathbf{4 b}(0.058 \mathrm{~g}, 80 \%)$ as a yellow solid.

${ }^{1} \mathrm{H}$ NMR (300 MHz, CDCl $), \delta(\mathrm{ppm}): 11.49(\mathrm{~s}, 1 \mathrm{H})$, 8.43 (d, $J=8.0 \mathrm{~Hz}, 1 \mathrm{H}), 7.68$ (t, $J=6.9 \mathrm{~Hz}, 1 \mathrm{H}$ ), 7.59-7.51 (m, 2H), 7.20 (d, $J=7.1 \mathrm{~Hz}, 1 \mathrm{H}), 6.58$ (d, $J$ $=7.1 \mathrm{~Hz}, 1 \mathrm{H})$.

${ }^{13} \mathrm{C}$ NMR, DEPT (75 MHz, $\left.\mathrm{CDCl}_{3}\right), \delta$ (ppm): 164.4 $(\mathrm{C}=\mathrm{O}), 138.1$ (C), $132.5(\mathrm{CH}), 127.6(\mathrm{CH}), 127.3$ (CH), $126.8(\mathrm{CH}), 126.2(\mathrm{CH}), 126.1(\mathrm{C}), 106.7(\mathrm{CH})$.

MS (EI, $70 \mathrm{eV}): \mathrm{m} / \mathrm{z}(\%)=145\left(\mathrm{M}^{+}, 100\right), 118(36)$, 90 (29).

HRMS-EI: $\mathrm{m} / \mathrm{z}$ calcd for $\mathrm{C}_{9} \mathrm{H}_{7} \mathrm{NO}\left[\mathrm{M}^{+}\right]$: 145.0528; found 145.0528 .

\section{2-Butylisoquinolin-1(2H)-one (4c)}

The general heterocyclization procedure was followed using 3c (0.100 g, $0.50 \mathrm{mmol}), \mathrm{CpRuCl}\left(\mathrm{PPh}_{3}\right)_{2}(0.036$ $\mathrm{g}, \quad 0.050 \mathrm{mmol})$ and pyridine $(3.30 \mathrm{~mL})$. Upon completion $(6 \mathrm{~h})$ and work-up, the residue was purified by flash column chromatography through silica gel using a 1:4 mixture of EtOAc/Hex as eluent to give $4 \mathrm{c}(0.074 \mathrm{~g}, 74 \%)$ as a brown oil.

${ }^{1} \mathrm{H}$ NMR (500 MHz, $\mathrm{CDCl}_{3}$ ), $\delta$ (ppm): 8.44 (d, $J=8.1$ $\mathrm{Hz}, 1 \mathrm{H}), 7.64-7.60(\mathrm{~m}, 1 \mathrm{H}), 7.51-7.46(\mathrm{~m}, 2 \mathrm{H}), 7.06$ $(\mathrm{d}, J=7.3 \mathrm{~Hz}, 1 \mathrm{H}), 6.48(\mathrm{~d}, J=7.3 \mathrm{~Hz}, 1 \mathrm{H}), 4.02-$ $3.98(\mathrm{~m}, 2 \mathrm{H}), 1.80-1.74(\mathrm{~m}, 2 \mathrm{H}), 1.40(\mathrm{td}, J=14.8$, $7.4 \mathrm{~Hz}, 2 \mathrm{H}), 0.96$ (t, $J=7.4 \mathrm{~Hz}, 3 \mathrm{H})$.

${ }^{13} \mathrm{C}$ NMR, DEPT (75 MHz, $\left.\mathrm{CDCl}_{3}\right), \delta$ (ppm): 162.1 $(\mathrm{C}=\mathrm{O}), 137.0(\mathrm{C}), 132.0(\mathrm{CH}), 131.7(\mathrm{CH}), 127.8$ $(\mathrm{CH}), 126.7(\mathrm{CH}), 126.3(\mathrm{C}), 125.8(\mathrm{CH}), 105.8(\mathrm{CH})$, $49.1\left(\mathrm{CH}_{2}\right), 31.4\left(\mathrm{CH}_{2}\right), 20.0\left(\mathrm{CH}_{2}\right), 13.8\left(\mathrm{CH}_{3}\right)$.

MS (EI, $70 \mathrm{eV}): \mathrm{m} / \mathrm{z}(\%)=202\left(\mathrm{M}^{+} 1,100\right), 149(8)$, 123 (16).

HRMS (ESI): $\mathrm{m} / \mathrm{z}$ calcd for $\mathrm{C}_{13} \mathrm{H}_{16} \mathrm{NO}\left[\mathrm{M}^{+}+1\right]$ : 202.1232; found: 202.1226.

\section{2-Butyl-2H-1,2-benzothiazine 1,1-dioxide (4d)}

The general heterocyclization procedure was followed using 3d (0.118 g, $0.50 \mathrm{mmol})$, $\mathrm{CpRuCl}\left(\mathrm{PPh}_{3}\right)_{2}(0.036$ g, $0.050 \mathrm{mmol})$ and pyridine $(3.30 \mathrm{~mL})$. Upon completion $(6 \mathrm{~h})$ and work-up, the residue was purified by flash column chromatography through silica gel using a 1:4 mixture of EtOAc/Hex as eluent to give $4 \mathbf{d}(0.072 \mathrm{~g}, 61 \%)$ as a brown oil.

${ }^{1} \mathrm{H}$ NMR (400 MHz, $\mathrm{CDCl}_{3}$ ), $\delta$ (ppm): 7.91 (d, $J=7.9$ $\mathrm{Hz}, 1 \mathrm{H}), 7.56$ (t, $J=7.6 \mathrm{~Hz}, 1 \mathrm{H}), 7.47-7.35(\mathrm{~m}, 2 \mathrm{H})$, $6.56(\mathrm{~d}, J=7.9 \mathrm{~Hz}, 1 \mathrm{H}), 6.24(\mathrm{~d}, J=7.9 \mathrm{~Hz}, 1 \mathrm{H})$, 3.76-3.71 (m, 2H), 1.75-1.67 (m, 2H), 1.35 (dd, $J=$ $15.1,7.5 \mathrm{~Hz}, 2 \mathrm{H}), 0.92(\mathrm{t}, J=7.5 \mathrm{~Hz}, 3 \mathrm{H})$.

${ }^{13} \mathrm{C}$ NMR, DEPT (75 MHz, $\left.\mathrm{CDCl}_{3}\right), \delta$ (ppm): 133.2 (C), $131.9(\mathrm{CH}), 131.3(\mathrm{CH}), 131.1(\mathrm{C}), 127.3(\mathrm{CH})$, $126.5(\mathrm{CH}), 121.6(\mathrm{CH}), 107.1(\mathrm{CH}), 48.0\left(\mathrm{CH}_{2}\right), 32.3$ $\left(\mathrm{CH}_{2}\right), 19.6\left(\mathrm{CH}_{2}\right), 13.6\left(\mathrm{CH}_{3}\right)$.

MS (CI): m/z (\%) = $238\left(\mathrm{M}^{+}+1,100\right), 182(6), 174$ (4).

HRMS (ESI): $\mathrm{m} / \mathrm{z}$ calcd for $\mathrm{C}_{12} \mathrm{H}_{16} \mathrm{NO}_{2} \mathrm{~S}\left[\mathrm{M}^{+}+1\right]$ : 238.0902; found: 238.0896 .

\section{1-(Isoquinolin-2(1H)-yl)ethanone (4f)}

The general heterocyclization procedure was followed using $3 f(0.086 \mathrm{~g}, 0.50 \mathrm{mmol})$, $\mathrm{CpRuCl}\left(\mathrm{PPh}_{3}\right)_{2}(0.036$ g, $0.050 \mathrm{mmol})$ and pyridine $(3.30 \mathrm{~mL})$. Upon completion $(5 \mathrm{~h})$ and work-up, the residue was purified by flash column chromatography through silica gel using a 1:4 mixture of EtOAc/Hex as eluent to give $\mathbf{4 f}(0.048 \mathrm{~g}, 56 \%)$ as a yellowish oil.

${ }^{1} \mathrm{H}$ NMR (250 MHz, $\mathrm{CDCl}_{3}$ ), $\delta$ (ppm): 7.20-7.04 (m, $4 \mathrm{H}), 6.65$ (d, $J=7.8 \mathrm{~Hz}, 1 \mathrm{H}), 5.82(\mathrm{~d}, J=7.8 \mathrm{~Hz}$, 1H), 4.94 (s, 2H), 2.20 (s, 3H).

${ }^{13} \mathrm{C}$ NMR, DEPT (75 MHz, $\left.\mathrm{CDCl}_{3}\right), \delta$ (ppm): 168.8 $(\mathrm{C}=\mathrm{O}), 130.4(\mathrm{C}), 129.4(\mathrm{C}), 127.6(\mathrm{CH}), 127.3(\mathrm{CH})$, $126.0(\mathrm{CH}), 125.9(\mathrm{CH}), 124.6(\mathrm{CH}), 109.6(\mathrm{CH})$, $44.3\left(\mathrm{CH}_{2}\right), 21.2\left(\mathrm{CH}_{3}\right)$. 
MS (CI): m/z (\%) = $174\left(\mathrm{M}^{+}+1,100\right), 132$ (30).

HRMS (ESI): $\mathrm{m} / \mathrm{z}$ calcd for $\mathrm{C}_{11} \mathrm{H}_{12} \mathrm{NO}\left[\mathrm{M}^{+}+1\right]$ : 174.0919; found: 174.0913 .

\section{2-Tosyl-1,2-dihydroisoquinoline (4g)}

The general heterocyclization procedure was followed using $3 g$ (0.142 g, $0.50 \mathrm{mmol}), \mathrm{CpRuCl}\left(\mathrm{PPh}_{3}\right)_{2}(0.036$ g, $0.050 \mathrm{mmol})$ and pyridine $(3.30 \mathrm{~mL})$. Upon completion $(4 \mathrm{~h})$ and work-up, the residue was purified by flash column chromatography through silica gel using a 1:4 mixture of EtOAc/Hex as eluent to give $\mathbf{4 g}(0.116 \mathrm{~g}, 82 \%)$ as a brown solid.

${ }^{1} \mathrm{H}$ NMR (250 MHz, $\left.\mathrm{CDCl}_{3}\right), \delta$ (ppm): 7.69 (d, $J=8.3$ $\mathrm{Hz}, 2 \mathrm{H}$ ), 7.27 (d, $J=8.0 \mathrm{~Hz}, 2 \mathrm{H}), 7.15-7.05$ (m, 2H), 6.98-6.90 (m, 2H), 6.76 (d, $J=7.8 \mathrm{~Hz}, 1 \mathrm{H}), 5.83$ (d, $J$ $=7.8 \mathrm{~Hz}, 1 \mathrm{H}), 4.56(\mathrm{~s}, 2 \mathrm{H}), 2.37(\mathrm{~s}, 3 \mathrm{H})$.

${ }^{13} \mathrm{C}$ NMR, DEPT (75 MHz, $\left.\mathrm{CDCl}_{3}\right), \delta$ (ppm): 144.1 (C), 134.4 (C), 130.4 (C), 129.8 (2 x CH), 128.0 (CH), $127.3(\mathrm{C}), 127.2(\mathrm{CH}), 127.1$ (2 x CH), $126.4(\mathrm{CH})$, $125.5(\mathrm{CH}), 124.4(\mathrm{CH}), 110.0(\mathrm{CH}), 47.1\left(\mathrm{CH}_{2}\right), 21.5$ $\left(\mathrm{CH}_{3}\right)$.

HRMS-EI: $\mathrm{m} / \mathrm{z}$ calcd for $\mathrm{C}_{16} \mathrm{H}_{15} \mathrm{NO}_{2} \mathrm{~S} \quad\left[\mathrm{M}^{+}\right]$: 285.0823; found: 285.0823 .

\section{1-Tosyl-1,4-dihydroquinoline (6)}

The general heterocyclization procedure was followed using 5 (0.142 g, $0.50 \mathrm{mmol})$, $\mathrm{CpRuCl}\left(\mathrm{PPh}_{3}\right)_{2}(0.036$ g, $0.050 \mathrm{mmol})$ and pyridine $(3.30 \mathrm{~mL})$. Upon completion (12 h) and work-up, the residue was purified by flash column chromatography through silica gel using a 1:4 mixture of EtOAc/Hex as eluent to give $6(0.085 \mathrm{~g}, 60 \%)$ as a brown oil.

${ }^{1} \mathrm{H}$ NMR (500 MHz, CDCl 3 ), $\delta$ (ppm): 7.81 (d, $J=8.2$ $\mathrm{Hz}, 1 \mathrm{H}), 7.44$ (d, $J=8.1 \mathrm{~Hz}, 2 \mathrm{H}), 7.26-7.22$ (m, 1H), 7.15 (d, $J=8.1 \mathrm{~Hz}, 3 \mathrm{H}), 6.89$ (d, $J=7.5 \mathrm{~Hz}, 1 \mathrm{H})$, 6.71 (d, $J=7.3 \mathrm{~Hz}, 1 \mathrm{H}), 5.54-5.49$ (m, 1H), 2.73 (d, $J$ $=3.6 \mathrm{~Hz}, 2 \mathrm{H}), 2.37$ (s, 3H).

${ }^{13} \mathrm{C}$ NMR, DEPT (75 MHz, $\left.\mathrm{CDCl}_{3}\right), \delta(\mathrm{ppm}): 143.9$ (C), 135.5 (C), 134.5 (C), 129.9 (C), 129.3 (2 x CH), $128.3(\mathrm{CH}), 127.6(\mathrm{CH}), 127.3(2 \times \mathrm{CH}), 126.8(\mathrm{CH})$, $126.2(\mathrm{CH}), 124.3(\mathrm{CH}), 116.6(\mathrm{CH}), 26.6\left(\mathrm{CH}_{2}\right), 21.6$ $\left(\mathrm{CH}_{3}\right)$.

MS (CI): m/z (\%) = $286\left(\mathrm{M}^{+}+1,85\right), 160$ (30), 132 (100).

HRMS (ESI): m/z calcd for $\mathrm{C}_{16} \mathrm{H}_{15} \mathrm{NO}_{2} \mathrm{SNa}\left[\mathrm{M}^{+}+\mathrm{Na}\right]$ : 308.0721; found: 308.0716.

\section{Acknowledgments}

We thank the MICINN [Project CTQ2011-28258, Consolider Ingenio 2010 (CSD2007-00006)], Xunta de Galicia and European Regional Development Fund (2007/XA084 and CN2011/054) for financial support. A. V.-F- thanks the USC for a predoctoral contract.
(1) Comprehensive Heterocyclic Chemistry III, (Eds. A. R. Katritzky, C. A. Ramsden, E. F. V. Scriven, R. J. K. Taylor), Elsevier, 2008.

(2) (a) Nakamura, I.; Yamamoto, Y. Chem. Rev. 2004, 104 2127. (b) D'Souza, D. M.; Mueller, T. J. J. Chem. Soc. Rev. 2007, 36, 1095.

(3) For reviews of metal vinylidenes in catalysis, see: (a) Bruce, M. I. Chem. Rev. 1991, 91, 197. (b) Bruneau, C.; Dixneuf, P. H. Acc. Chem. Res. 1999, 32, 311. (c) Trost, B. M. Acc. Chem. Res. 2002, 35, 695. (d) Bruneau, C.; Dixneuf, P. H. Angew. Chem., Int. Ed. 2006, 45, 2176. (e) Trost, B. M.; McClory, A. Chem. Asian J. 2008, 3, 164.

(4) McDonald, F. E.; Reddy, K. S.; Diaz, Y. J. Am. Chem. Soc. 2000, 122, 4304 and references therein.

(5) (a) Trost, B. M.; Rhee, Y. H. J. Am. Chem. Soc. 1999, 121, 11680. (b) Trost, B. M.; Rhee, Y. H. J. Am. Chem. Soc. 2002, 124, 2528.

(6) Trost, B. M.; Rhee, Y. H. J. Am. Chem. Soc. 2003, 125, 7482.

(7) (a) Alcazar, E.; Pletcher, J. M.; McDonald, F. E. Org. Lett. 2004, 6, 3877. (b) Koo, B.; McDonald, F. E. Org. Lett. 2007, 9, 1737.

(8) Varela-Fernández, A.; González-Rodríguez, C.; Varela, J. A.; Castedo, L.; Saá, C. Org. Lett. 2009, 11, 5350.

(9) Varela-Fernández, A.; García-Yebra, C.; Varela, J. A.; Esteruelas, M. A.; Saá, C. Angew. Chem., Int. Ed. 2010, 49, 4278.

(10) McDonald, F. E.; Chatterjee, A. K. Tetrahedron Lett. 1997, 38, 7687.

(11) Trost, B. M.; McClory, A. Angew. Chem., Int. Ed. 2007, 46, 2074.

(12) Nair, R. N.; Lee, P. J.; Rheingold, A. L.; Grotjahn, D. B. Chem. Eur. J. 2010, 16, 7992

(13) Varela-Fernández, A.; Varela, J. A.; Saá, C. Adv. Synth. Catal. 2011, 353, 1933.

\section{References}


